\title{
Segregated quantum phases of dipolar bosonic mixtures in two-dimensional optical lattices
}

\author{
Rukmani Bai, ${ }^{1,2,3}$ Deepak Gaur, ${ }^{1,2}$ Hrushikesh Sable, ${ }^{1,2}$ Soumik Bandyopadhyay, ${ }^{1,2}$ K. Suthar, ${ }^{1,4}$ and D. Angom ${ }^{1}$ \\ ${ }^{1}$ Physical Research Laboratory, Ahmedabad - 380009, Gujarat, India \\ ${ }^{2}$ Indian Institute of Technology Gandhinagar, Palaj, Gandhinagar - 382355, Gujarat, India \\ ${ }^{3}$ Institute for Theoretical Physics III and Center for Integrated Quantum Science and Technology, \\ University of Stuttgart, 70550 Stuttgart, Germany \\ ${ }^{4}$ Institute of Theoretical Physics, Jagiellonian University in Kraków, Lojasiewicza 11, 30-348 Kraków, Poland
}

\begin{abstract}
We identify the quantum phases in a binary mixture of dipolar bosons in two-dimensional optical lattices. Our study is motivated by the recent experimental realization of binary dipolar condensate mixtures of Er-Dy [Phys. Rev. Lett. 121, 213601 (2018)]. We model the system by using the extended two-species Bose-Hubbard model and calculate the ground-state phase diagrams by using mean-field theory. For selected cases we also obtain analytical phase boundaries by using the site-decoupled mean-field theory. For comparison we also examine the phase diagram of two-species Bose-Hubbard model. Our results show that the quantum phases with the long-range intraspecies interaction phase separate with no phase ordering. The introduction of the long-range interspecies interaction modifies the quantum phases of the system. It leads to the emergence of phase-separated quantum phases with phase ordering. The transition from the phase-separated quantum phases without phase ordering to phase ordered ones breaks the inversion symmetry.
\end{abstract}

\section{INTRODUCTION}

The Bose-Hubbard model [1, 2] describes the physics of ultracold bosonic atoms trapped in optical lattices [3]. The variation of the hopping term, equivalent to kinetic terms in continuum models, in the Bose-Hubbard model drives a quantum phase transition from the Mott insulator (MI) to the superfluid (SF) phase. And this transition has been experimentally observed [4]. The interparticle interaction in the BoseHubbard model is onsite or contact in nature. The introduction of the nearest neighbor $(\mathrm{NN})$ interaction in the Bose-Hubbard model generates two more phases: density wave (DW) and supersolid (SS). This model with the NN interactions is referred to as the extended Bose-Hubbard model [5] and shows rich physics compared with the Bose-Hubbard model. Such a model captures the physics of dipolar ultracold quantum gases in optical lattices $[6,7]$. A more complex system, ideal to model several condensed-matter systems, is to fill the optical lattice with two species Bose-Einstein Condensate or binary condensate. A binary condensate could be a condensate mixture of two different atomic species [8-13], two hyperfine states of an atom [14-23] or two different isotopes of an atomic species [24-26]. It was experimentally first realized in the two hyperfine states $\left|F=2, m_{F}=2\right\rangle$ and $\left|F=1, m_{F}=-1\right\rangle$ of ${ }^{87} \mathrm{Rb}$ atom [14]. The binary condensates, in the weakly interacting continuum systems, have been used to investigate novel phenomena such as pattern formation [27-33], phase separation [10, 12, 13, 23, 24, 34-38], nonlinear dynamical excitations [21,39-44], collective excitations [45-51], Kibble-Zurek mechanism [52], and the production of dipolar molecules [53-55]. The phase separation, among all the phenomena is a unique property of binary condensates. In this work we study the binary condensates trapped in the optical lattices that can be described by the Bose-Hubbard model with appropriate modifications. The experimental realization in optical lattices are reported in Refs. [56, 57] and early theoretical studies are presented in Refs. [58-61]. A remarkable recent achievement related to binary condensates is the experimental realization with dipolar quantum mixtures of Er-Dy, reported in a recent work [62].

The physics of the two-species Bose-Hubbard model (TBHM), the lattice counterpart of a binary condensate, in one dimension has been investigated in detail [63-65]. And, there has been some works in two dimension as well [58-61, 6669]. The phase diagram of TBHM shows different combinations of mixed MI-SF phases apart from the Mott insulator and superfluid phases. And, these have been investigated by using quantum Monte Carlo [60, 61], mapping to spin systems [58], and with mean-field theory [59, 66-68, 70]. These studies, except for Ref. [70], considered homogeneous systems. However, hitherto the phenomenon of phase separation in two-dimensional TBHM has yet to be investigated in detail.

The quantum phases of TBHM in the phase-separated domain, unlike in the binary condensates, do not show segregation into two spatial domains. We attribute this to the lack of long-range interactions. The simplest modification to include the effect of long-range interactions is to add nearest neighbor interactions. The extended Bose-Hubbard model, as mentioned earlier, supports two more quantum phases: density wave [71-73] and supersolid [73-78]. The density wave phase is an insulating phase similar to the Mott insulator phase but it has crystalline order or diagonal long-range order. And, the supersolid phase is a compressible phase with both diagonal and off-diagonal long-range order. In a recent study of extended TBHM (eTBHM) [79], it was shown that the supersolid phase exists for small value of $\mathrm{NN}$ interactions. In this work, the NN interaction was limited to either one of the species or between the species. We address this research gap by including all the possible intra- and interspecies NN interactions. Such a model is apt to describe the physics of dipolar Bose-Bose mixtures in optical lattices. An example of such a combination is the recently realized Er-Dy mixture [62]. An important result of our work is the possibility to realize compressible and incompressible quantum phases with spatial segregation. Such a phase could be instrumental in examining superfluid instabilities and other nonequilibrium properties in the lattice models of quantum liquids.

The remainder of the paper is organized into four sections. 
In Sec. II we describe the zero-temperature Hamiltonian of the TBHM and discuss the Gutzwiller mean-field theory of the model. We then discuss the mean-field decoupling theory to calculate the compressible-incompressible phase boundaries analytically. This is followed by a brief discussion on the characterization of quantum phases. The phase diagrams of TBHM are discussed in the Sec. III. Section IV includes a discussion on the phase diagram of the eTBHM. In particular, the miscible and immiscible phases. We also check the dynamical stability of the quantum phases by computing the collective excitations of the system. We end the paper with conclusion in Sec. V.

\section{THEORY}

\section{A. Two-species Bose-Hubbard model Hamiltonian}

At zero temperature, the TBHM Hamiltonian, which describes the physics of a binary condensate in a twodimensional optical lattice, is [80]

$$
\begin{aligned}
\hat{H}^{\mathrm{TBH}}= & -\sum_{p, q, k}\left[\left(J_{x}^{k} \hat{b}_{p+1, q}^{\dagger k} \hat{b}_{p, q}^{k}+\text { H.c. }\right)+\left(J_{y}^{k} \hat{b}_{p, q+1}^{\dagger k} \hat{b}_{p, q}^{k}\right.\right. \\
& \left.+ \text { H.c. })-\frac{U_{k k}}{2} \hat{n}_{p, q}^{k}\left(\hat{n}_{p, q}^{k}-1\right)+\tilde{\mu}_{p, q}^{k} \hat{n}_{p, q}^{k}\right] \\
& +\sum_{p, q} U_{12} \hat{n}_{p, q}^{1} \hat{n}_{p, q}^{2}
\end{aligned}
$$

where $k=1,2$ is the species index, $(p, q)$ are the lattice indices, $J_{x}^{k}\left(J_{y}^{k}\right)$ is the NN hopping strength along $x(y)$ directions, $\hat{b}_{p, q}^{\dagger k}\left(\hat{b}_{p, q}^{k}\right)$ is the creation (annihilation) operator, and $\hat{n}_{p, q}^{k}$ is the number operator at site $(p, q) . U_{k k}$ is intraspecies interaction strength, and $U_{12}$ is the interspecies interaction strength between two species. Furthermore, $\tilde{\mu}_{p, q}^{k}=\mu^{k}-\varepsilon_{p, q}^{k}$, is the local chemical potential at each site for the two species where $\varepsilon_{p, q}^{k}$ is the envelop potential for the species. For a system of $K \times L$ lattices sites, the index along $x(y)$ has values $p=1, \ldots K(q=1, \ldots L)$. The unique feature of the binary condensates is the phase separation and for continuum systems, the criterion for phase segregation is $U_{12}^{2}>U_{11} U_{22}$ $[34,81]$. Otherwise, it is in the miscible phase. For the case of strongly interacting binary condensates in optical lattices, described by the above Hamiltonian, we show the existence of different phases in both the miscible and immiscible domains.

To obtain the ground state of the Hamiltonian in Eq. (1), we use single-site Gutzwiller mean-field (SGMF) theory [7, 82-86]. The starting point of this theory is to separate the operators into mean-field and fluctuation operator components as $\hat{b}_{p, q}^{k}=\phi_{p, q}^{k}+\delta \hat{b}_{p, q}^{k}$ and $\hat{b}_{p, q}^{\dagger k}=\phi_{p, q}^{k *}+\delta \hat{b}_{p, q}^{\dagger k}$. Then, the Hamiltonian in Eq. (1) is reduced to the sum of the single-site mean-field Hamiltonian

$$
\begin{aligned}
\hat{h}_{p, q}^{\mathrm{TBH}}= & -\sum_{k}\left[J_{x}^{k}\left(\hat{b}_{p+1, q}^{\dagger k} \phi_{p, q}^{k}+\phi_{p+1, q}^{k *} \hat{b}_{p, q}^{k}\right)+\right.\text { H.c. } \\
& +J_{y}^{k}\left(\hat{b}_{p, q+1}^{\dagger k} \phi_{p, q}^{k}+\phi_{p, q+1}^{k *} \hat{b}_{p, q}^{k}\right)+\text { H.c. } \\
& \left.-\frac{U_{k k}}{2} \hat{n}_{p, q}^{k}\left(\hat{n}_{p, q}^{k}-1\right)+\tilde{\mu}_{p, q}^{k} \hat{n}_{p, q}^{k}\right]+U_{12} \hat{n}_{p, q}^{1} \hat{n}_{p, q}^{2}
\end{aligned}
$$

where $\phi_{p, q}^{k}\left(\phi_{p, q}^{k *}\right)$ is the superfluid order parameter. With this definition of the single-site mean-field Hamiltonian, the total Hamiltonian of the system is

$$
\hat{H}^{\mathrm{TBH}}=\sum_{p, q} \hat{h}_{p, q}^{\mathrm{TBH}} .
$$

For the details of the derivations, see Ref. [84]. To get the ground state we diagonalize the Hamiltonian in Eq. (2) at each site. And, for this we use the Gutzwiller ansatz, based on which the ground state at site $(p, q)$ is [68]

$$
|\psi\rangle_{p, q}=\sum_{n_{1}, n_{2}} c_{n_{1}, n_{2}}^{(p, q)}\left|n_{1}, n_{2}\right\rangle_{p, q} .
$$

Here, $\left|n_{1}, n_{2}\right\rangle$ is a Fock state, which is the direct product of the $n_{1}$ and $n_{2}$ occupation number states of the first and second species, respectively. The occupation number states $n_{k} \in\left[0, N_{b}-1\right]$, where $N_{b}$ is the total number of local Fock states used in the computation, and $c_{n_{1}, n_{2}}^{p, q}$ are complex coefficients with $\sum_{n_{1}, n_{2}}\left|c_{n_{1}, n_{2}}^{(p, q)}\right|^{2}=1$. From the ground state, we can compute the new superfluid order parameter of the two species as

$$
\begin{aligned}
& \phi_{p, q}^{1}={ }_{p, q}\left\langle\psi\left|\hat{b}_{p, q}^{1}\right| \psi\right\rangle_{p, q}=\sum_{n_{1}, n_{2}} \sqrt{n_{1}} c_{n_{1}-1, n_{2}}^{(p, q) *} c_{n_{1}, n_{2}}^{(p, q)}, \\
& \phi_{p, q}^{2}={ }_{p, q}\left\langle\psi\left|\hat{b}_{p, q}^{2}\right| \psi\right\rangle_{p, q}=\sum_{n_{1}, n_{2}} \sqrt{n_{2}} c_{n_{1}, n_{2}-1}^{(p, q) *} c_{n_{1}, n_{2}}^{(p, q)} .
\end{aligned}
$$

Similarly, corresponding lattice occupancies are

$$
\begin{aligned}
& \rho_{p, q}^{1}={ }_{p, q}\left\langle\psi\left|\hat{n}_{p, q}^{1}\right| \psi\right\rangle_{p, q}=\sum_{n_{1}, n_{2}} n_{1}\left|c_{n_{1}, n_{2}}^{(p, q)}\right|^{2}, \\
& \rho_{p, q}^{2}={ }_{p, q}\left\langle\psi\left|\hat{n}_{p, q}^{2}\right| \psi\right\rangle_{p, q}=\sum_{n_{1}, n_{2}} n_{2}\left|c_{n_{1}, n_{2}}^{(p, q)}\right|^{2} .
\end{aligned}
$$

Using the new superfluid order parameters, the ground state of the next lattice site is computed and this process is repeated until all the lattices sites are covered. One such sweep is identified as an iteration and we then, start the process again for the next iteration. The iterations are carried out until the convergence criterion $\left|\phi_{p, q}^{n-1}-\phi_{p, q}^{n}\right| \lesssim 10^{-12}$ is satisfied at the $n^{\text {th }}$ iteration. In the present work, to determine the phase diagrams, we consider lattice system of size $10 \times 10$ and choose $N_{b}=7$. That is, $K$ and $L$ are both 10 . We find that the phase boundaries remain unchanged when the system size is augmented to $20 \times 20$. We also use the augmented system size to validate key findings. In addition, we employ periodic boundary conditions to model an infinite-sized system. 


\section{B. Extended two-species Bose-Hubbard model Hamiltonian}

The Bose-Hubbard model with NN interaction, referred to as the extended Bose-Hubbard model, exhibits a richer phase diagram than does the Bose-Hubbard model and it has the novel feature of harbouring the supersolid phase. The phase diagram of this model consists of density wave, supersolid, Mott insulator and superfluid phases. Similarly, the eTBHM also exhibits these phases as well as miscible and segregated phases and the model Hamiltonian of the system is

$$
\begin{aligned}
\hat{H}^{\mathrm{ext}}= & \hat{H}^{\mathrm{TBH}}+\sum_{p, q, k}\left[V _ { k } \hat { n } _ { p , q } ^ { k } \left(\hat{n}_{p-1, q}^{k}+\hat{n}_{p+1, q}^{k}+\hat{n}_{p, q-1}^{k}\right.\right. \\
& \left.+\hat{n}_{p, q+1}^{k}\right)+V_{12} \hat{n}_{p, q}^{k}\left(\hat{n}_{p-1, q}^{3-k}+\hat{n}_{p+1, q}^{3-k}+\hat{n}_{p, q-1}^{3-k}\right. \\
& \left.\left.+\hat{n}_{p, q+1}^{3-k}\right)\right]
\end{aligned}
$$

here $V_{k}$ and $V_{12}$ are the intraspecies and interspecies NN interaction strengths respectively. In the experiments the ratio of $\mathrm{NN}$ interaction to the on-site interaction can be varied by tuning the on-site interaction through a magnetic Feshbach resonance. The NN interaction, arising from the dipole-dipole interaction, can also be varied by rotation of the dipoles with a time-dependent external magnetic field [87, 88]. Using this method it can even be turned off. The quantum phases obtained from the model described by the above Hamiltonian are relevant to the experimental realizations with the dipoles oriented perpendicular to the lattice plane. In a latter section, Section IV B 3, we provide a brief description of the quantum phases when the tilt angle $\theta$ is nonzero. Here, $\theta$ is the angle between the orientation of the dipoles and the normal to the lattice plane. Thus, to relate with the experimental observations and predict possible phases we vary the inter- and intraspecies interaction strengths. We use SGMF theory to obtain the ground state of the system, then, in this method the total Hamiltonian is the sum of the single-site mean-field Hamiltonian

$$
\begin{aligned}
\hat{h}_{p, q}^{\mathrm{ext}}= & \hat{h}_{p, q}^{\mathrm{TBH}}+\sum_{k}\left[V _ { k } \hat { n } _ { p , q } ^ { k } \left(\left\langle\hat{n}_{p-1, q}^{k}\right\rangle+\left\langle\hat{n}_{p+1, q}^{k}\right\rangle+\left\langle\hat{n}_{p, q-1}^{k}\right\rangle\right.\right. \\
& \left.+\left\langle\hat{n}_{p, q+1}^{k}\right\rangle\right)+V_{12} \hat{n}_{p, q}^{k}\left(\left\langle\hat{n}_{p-1, q}^{3-k}\right\rangle+\left\langle\hat{n}_{p+1, q}^{3-k}\right\rangle+\left\langle\hat{n}_{p, q-1}^{3-k}\right\rangle\right. \\
& \left.\left.+\left\langle\hat{n}_{p, q+1}^{3-k}\right\rangle\right)\right] .
\end{aligned}
$$

We diagonalize this Hamiltonian at each site separately, and obtain the ground state. The NN-interaction term contributes to the diagonal matrix element. From the single-site wavefunction, the superfluid order parameter and lattice occupancy can be calculated from the expressions in Eqns. (5a), (5b) and (6a), (6b).

\section{Mean-field decoupling theory}

\section{Two-species Bose-Hubbard model}

To calculate the phase boundaries between Mott insulator and superfluid phases analytically we use the site decoupled mean-field theory [7, 89, 90]. For this, we adapt perturbative analysis of the mean-field Hamiltonian in Eq. (2). It is important to note that the superfluid order parameter $\phi_{p, q}^{k}$ is zero in the Mott insulator phase, but nonzero in the superfluid phase. So, the vanishing of the superfluid order parameter $\phi_{p, q}^{k} \rightarrow 0^{+}$ marks the MI-SF phase boundary in the phase diagram. With this consideration, in the site-decoupled mean-field theory, the interaction and the chemical potential terms constitute the unperturbed Hamiltonian $\hat{h}_{p, q, 0}^{\mathrm{TBH}}$. From Eq. (2),

$$
\begin{aligned}
\hat{h}_{p, q, 0}^{\mathrm{TBH}}= & \sum_{k}\left[\frac{U_{k k}}{2} \hat{n}_{p, q}^{k}\left(\hat{n}_{p, q}^{k}-1\right)-\tilde{\mu}_{p, q}^{k} \hat{n}_{p, q}^{k}\right] \\
& +U_{12} \hat{n}_{p, q}^{1} \hat{n}_{p, q}^{2},
\end{aligned}
$$

which is diagonal with respect to the Fock basis states. Then, the hopping terms in Eq. (2) act as the perturbation,

$$
\begin{aligned}
\hat{h}_{p, q, 1}^{\mathrm{TBH}}= & -\sum_{k}\left[J_{x}^{k}\left(\hat{b}_{p+1, q}^{\dagger k} \phi_{p, q}^{k}+\phi_{p+1, q}^{k *} \hat{b}_{p, q}^{k}\right)+\right.\text { H.c. } \\
& \left.+J_{y}^{k}\left(\hat{b}_{p, q+1}^{\dagger k} \phi_{p, q}^{k}+\phi_{p, q+1}^{k *} \hat{b}_{p, q}^{k}\right)+\text { H.c. }\right]
\end{aligned}
$$

with the superfluid order parameter $\phi_{p, q}^{k}$ as the perturbation parameter. Then, from the first-order perturbative correction to the ground-state wavefunction (details given in Appendix A), we have

$$
\phi_{p, q}^{k}=J \bar{\phi}_{p, q}^{k}\left(\frac{n_{p, q}^{k}+1}{n_{p, q}^{k} U-\bar{\mu}_{p, q}^{k}}-\frac{n_{p, q}^{k}}{\left(n_{p, q}^{k}-1\right) U-\bar{\mu}_{p, q}^{k}}\right),
$$

with

$$
\begin{aligned}
\bar{\mu}_{p, q}^{k} & =\tilde{\mu}_{p, q}^{k}-U_{12} n_{p, q}^{3-k}, \\
\bar{\phi}_{p, q}^{k} & =\left(\phi_{p+1, q}^{k}+\phi_{p-1, q}^{k}+\phi_{p, q+1}^{k}+\phi_{p, q-1}^{k}\right) .
\end{aligned}
$$

For a homogeneous lattice system $\varepsilon_{p, q}^{k}=0$. Then, in the Mott insulator phase the total density $\rho=\rho^{1}+\rho^{2}$ is integer commensurate and $\phi_{p, q}^{k}=0$. In the superfluid phase, the order parameter is nonzero and uniform, say $\phi_{p, q}^{k}=\varphi_{0}^{k}$. With these considerations, $\bar{\phi}_{p, q}^{k}=\bar{\phi}^{k}=4 \varphi_{0}^{k}$. Starting from the superfluid phase, at the SF-MI phase boundary $\varphi_{0}^{k} \rightarrow 0^{+}$. Considering this limit in Eq. (11), we obtain the equation which defines the phase boundary in terms of $J$ for a particular value of $\mu$.

For the $\rho=2$ Mott lobe, in the miscible domain, atoms of both the species fill all the lattice sites. That is, $n_{p, q}^{1}=n_{p, q}^{2}=$ 1. The MI-SF phase boundary is, then, defined by

$$
\frac{1}{4 J}=\frac{2}{U-\mu+U_{12}}+\frac{1}{\mu-U_{12}} .
$$

On the other hand for finite $U_{12}$, the system is in the immiscible domain for the $\rho=1$ Mott lobe. The density pattern has 
one atom at each lattice site chosen randomly from the two species. Thus, at a given lattice site $(p, q)$ we can have the occupancies as $n_{p, q}^{1}=1, n_{p, q}^{2}=0$ or $n_{p, q}^{1}=0, n_{p, q}^{2}=1$. In the perturbative analysis, without loss of generality, we consider neighboring lattice sites which are occupied by atoms of different species. This is also one realization of the energetically favourable configuration for $U_{12}<U$. Then, with the correction arising from $b^{\dagger 1} \phi^{1}$, the equation

$$
\frac{1}{4 J}=\frac{2}{U_{12}-\mu}+\frac{1}{\mu},
$$

defines the phase boundary of the Mott lobe with $\rho=1$. Based on similar analysis, we can obtain the phase boundary of other Mott lobes. For which we have to use Eqs.(12) and (13) for the even and odd-integer values of $\rho$, respectively.

\section{Extended two-species Bose-Hubbard model}

We extend the analysis done in previous section to the eTBHM case. The expression of the order parameter is similar to Eq. (11) but $\bar{\mu}_{p, q}^{k}$ is given by

$$
\bar{\mu}_{p, q}^{k}=\tilde{\mu}_{p, q}^{k}-U_{12} n_{p, q}^{3-k}-4 V_{k} n_{p, q}^{k}-4 V_{12} n_{p, q}^{3-k},
$$

For the $\operatorname{MI}(1,1)$ phase with $\rho=2$, the occupancies are $n_{p, q}^{k}=$ 1. Furthermore, assuming $V_{1}=V_{2}$, the MI-SF boundary is given by

$$
\frac{1}{4 J}=\frac{2}{U-\bar{\mu}}+\frac{1}{\bar{\mu}} .
$$

with $\bar{\mu}=\mu-U_{12}-4 V_{1}-4 V_{12}$. Similarly, the phase boundary for the $\operatorname{MI}(2,2)$ lobe can be obtained by choosing $n_{p, q}^{k}=2$ in Eq.(11) with $\bar{\mu}_{p, q}^{k}$ given by Eq.(14). In the density wave phase, the two sublattice structure description is applicable. Using this, the density wave to compressible phase boundary for $V_{1}=V_{2}$ and $V_{12}=0$ is given by

$$
\begin{aligned}
\frac{1}{16 J^{2}}= & \left\{\frac{n^{1, B}+1}{U n^{1, B}-\mu+U_{12} n^{2, B}+4 V_{1} n^{1, A}}\right. \\
& \left.-\frac{n^{1, B}}{U\left(n^{1, B}-1\right)-\mu+U_{12} n^{2, B}+4 V_{1} n^{1, A}}\right\} \\
& \times\left\{\frac{n^{1, A}+1}{U n^{1, A}-\mu+U_{12} n^{2, A}+4 V_{1} n^{1, B}}\right. \\
& \left.-\frac{n^{1, A}}{U\left(n^{1, A}-1\right)-\mu+U_{12} n^{2, A}+4 V_{1} n^{1, B}}\right\}
\end{aligned}
$$

The details are given in Appendix B. As an example consider the $\mathrm{DW}(1,0)$ phase. It has $n^{1, A}=1, n^{1, B}=0, n^{2, A}=$ 0 and $n^{2, B}=1$. From the above equation, the $\operatorname{DW}(1,0)-$ compressible phase boundary is given by

$$
\frac{1}{16 J^{2}}=\left\{\frac{1}{-\mu+U_{12}+4 V_{1}}\right\} \times\left\{\frac{2}{U-\mu}+\frac{1}{\mu}\right\} .
$$

Using Eq. (16), we can also calculate the phase boundaries for other density wave phases.

\section{Characterization of the phases}

\begin{tabular}{lllll}
\hline \hline Quantum phase & $\rho$ & $\phi$ & $\Delta \rho^{k}$ & $\Delta \phi^{k}$ \\
\hline Mott Insulator & Integer & $=0$ & $=0$ & $=0$ \\
Superfluid & Real & $\neq 0$ & $=0$ & $=0$ \\
Density Wave & Integer & $=0$ & $\neq 0$ & $=0$ \\
Supersolid & Real & $\neq 0$ & $\neq 0$ & $\neq 0$ \\
\hline \hline
\end{tabular}

TABLE I. Classification of different quantum phases with order parameters for our systems.

To identify different quantum phases of the system we compute the density contrast $\Delta \rho^{k}$, order parameter contrast $\Delta \phi^{k}$ and compressibility $\kappa^{k}$. To define $\Delta \rho^{k}$, divide the lattice site occupancies as

$$
n_{p, q}^{k}= \begin{cases}n^{k, A} & \text { if }(p, q) \in \text { sublattice A } \\ n^{k, B} & \text { if }(p, q) \in \text { sublattice B }\end{cases}
$$

then, the density contrast of the $k$ th species is

$$
\Delta \rho^{k}=n^{k, A}-n^{k, B} .
$$

The order parameter contrast is defined similarly as

$$
\Delta \phi^{k}=\phi^{k, A}-\phi^{k, B}
$$

where $\phi^{k, A}$ and $\phi^{k, B}$, like in the case of density are the values of the order parameters at lattice sites with $(p, q)$ belonging to sublattices A and B, respectively. The compressibility of each species are calculated by using the definition $\partial \mu^{k} / \partial \rho^{k}$.

The TBHM, like the single species Bose-Hubbard model, shows two phases, Mott insulator and superfluid. The Mott insulator phase is an incompressible phase with integer commensurate density $n^{k, A}=n^{k, B} \in \mathbb{N}$. And incompressibility implies zero superfluid order parameter $\phi^{k, A}=\phi^{k, B}=0$. The superfluid phase, on the other hand is compressible. Hence, it has $n^{k, A}=n^{k, B} \in \mathbb{R}, \phi^{k, A}=\phi^{k, B} \in \mathbb{R}$ and $\kappa^{k} \neq 0$. For these two phases, the density and superfluid order parameters are uniform, so the contrast order parameters $\Delta \rho^{k}$ and $\Delta \phi^{k}$ are zero. In the eTBHM, the NN interaction leads to the emergence of two more quantum phases, density wave and supersolid. These two phases have nonuniform density and superfluid order parameters. As a result the distinguishing features of these phases are nonzero contrast order parameters. The density wave phase has integer $n_{p, q}^{k}$ with $n^{k, A} \neq n^{k, B}$ and $\Delta \rho^{k} \in \mathbb{N}$. This phase has zero superfluid order parameter $\phi^{k, A}=\phi^{k, B}=0$ and hence, is incompressible. The supersolid phase has real $n_{p, q}^{k}$ with $n^{k, A} \neq n^{k, B}$ and $\Delta \rho^{k} \in \mathbb{R}$. The superfluid order parameter in this phase is nonzero and nonuniform. This implies that $n^{k, A} \neq n^{k, B}$ and $\phi^{k, A} \neq \phi^{k, B}$. So, both the contrast order parameters are nonzero in this phase. For easy reference the properties of the different quantum phases are listed in Table. I. 


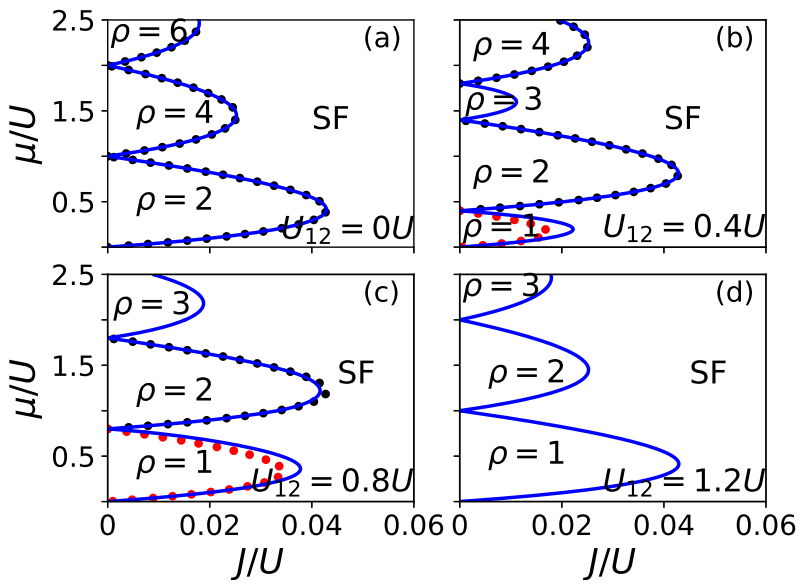

FIG. 1. Phase diagram of TBHM by varying the interspecies interaction strength $U_{12}$. Blue solid lines represent numerically obtained phase boundaries for the mean field Hamiltonian. Filled dots marks phase boundaries between compressible and incompressible phases, obtained analytically by perturbative analysis of the meanfield Hamiltonian. The odd occupancy Mott lobes appear for nonzero $U_{12}$ and enlarges with increasing $U_{12}$.

\section{PHASE DIAGRAM OF TWO-SPECIES BOSE-HUBBARD MODEL}

To compute the ground-state wavefunction and determine the phase, we initialize the superfluid order parameter $\phi$. This, then, defines the Hamiltonian in Eq. (2) and Hamiltonian matrix elements are computed by using the Gutzwiller wavefunction in Eq. (4). By diagonalizing the Hamiltonian matrix for each site we obtain the ground-state wavefunction. From the results, the MI-SF phase boundary is identified based on the superfluid order parameter and the lattice occupancy. For the incompressible Mott insulator phase, at each lattice site, $\phi$ is zero and $\rho$ is integer commensurate. For the superfluid phase, $\phi$ is nonzero and $\rho$ is real commensurate. The phase diagrams of TBHM given in Eq. (2) for different values of $U_{12}$ are shown in Fig. 1.

For simplicity, we consider symmetric hopping $J_{x}^{k}=J_{y}^{k}=$ $J$, equal chemical potential $\tilde{\mu}_{p, q}^{1}=\tilde{\mu}_{p, q}^{2}=\mu$ and identical intraspecies interactions $U_{k k}=U$. We scale all the energies with $U$, and define the phase diagram in the $J / U-\mu / U$ plane.

\section{A. Zero temperature phase diagram}

The phase diagram consists of a sequence of Mott lobes having integer $\rho$. Without the interspecies interaction $U_{12}=$ 0 , as shown in Fig. 1(a), the phase diagram is equivalent to the case of single species, but with twice the occupancy. That is the Mott lobes, which have $\rho=2 n$ with $\rho^{1}=\rho^{2}=n$ and $n \in \mathbb{N}$. So, the lowest Mott lobe has $\rho=2$ and each lattice has one atom from each of the two species. As a result, the phase diagram is identical to the single species case. With the introduction of the interspecies interaction $\left(U_{12} \neq 0\right)$ the half filled lobes like $\rho=1$ emerge in the phase diagram with $0<\rho^{1}<1$, and then, $\rho^{2}=1-\rho^{1}$. This is discernible for $U_{12}=0.4 U$ from the Fig. 1(b). Based on the form of the interactions in the Hamiltonian of the system, the energies of system is degenerate for all the possible combinations of $\rho^{1}$ and $\rho^{2}$ in the allowed ranges. For example, with $U_{12}=0.4 U$ and for $\mu / U=0.2, J / U=0.01$ we observe $0.33 \lesssim \rho^{1} \lesssim$ 0.7 . In the figure, the half filling lobe $\rho^{k}=0.5$ and $\rho=1$ at $J / U=0$ lies in the domain $0 \leqslant \mu / U \leqslant 0.4$. In general, in the miscible domain, the half filling lobe $\rho=1$ at $J / U=0$ lies in the domain $0 \leqslant \mu / U \leqslant U_{12} / U$. The other Mott lobes with higher $\rho$ occur at the higher values $\mu / U$. In general, the Mott lobes have $\rho=n$ with $n \in \mathbb{N}$ and $\rho^{k}=n / 2$. Thus, for Mott lobes with odd $n$ the average occupancy of each species is half integer.

With increasing $U_{12}$, the Mott lobes with odd-integer occupancies grows in size, but the size of the lobes with eveninteger occupancies remains the same until $U_{12}=U_{k k}$ but shifts to higher $\mu / U$. This can be understood from Eq. (11). The trend is discernible from the phase diagrams in Fig.1(b)1(c). This, in the case of weakly interacting binary condensates, is equivalent to a march towards phase separation [3438]. For $U_{12}>U_{k k}$, the criterion for phase separation, the size of the Mott lobe $\rho=2$ is different. But, once the phase separation criterion is met, there is no change in the phase diagram with further increase in $U_{12}$. As an example the phase diagram for $U_{12}=1.2 U$ is shown in Fig.1(d). The lobes in this phase diagram are the same as in Fig.1(a). The only difference is the occupancy is $\rho=n$ with $n \in \mathbb{N}$ and $\rho^{k}=n / 2$. As a result, the density pattern of the lowest Mott lobe $(\rho=1)$ has one atom at each lattice site chosen randomly from the two species. The important point is that the Mott lobes have the same sizes for $U_{12}=0$ and $U_{12} \geqslant U_{k k}$. But, the occupancy and hence the density patterns are different.

To verify our results we do a comparison with quantum Monte Carlo results reported in earlier works [91, 92]. For this, we check the order of the MI-SF quantum phase transition of the $\rho=2$ Mott lobe. As a measure we compute the energy per particle for fixed $\mu$ and find that the transition is first order close to the tip of the Mott lobe. And, it is second order away from the tip. This is consistent with the quantum Monte Carlo results [91, 92]. To assess the impact of the quantum fluctuations on the nature of the phase transitions, we employ cluster-Gutzwiller Mean Field (CGMF) theory. This is a multisite generalization of the SGMF theory, and captures the quantum correlations accurately within each cluster. We refer to Refs. [84, 85, 93] for the details. In the present case, we repeat the SGMF computations by using $2 \times 2$ clusters, which is sufficient to probe the effects of quantum fluctuations. With the CGMF method, apart from the enhancement of the Mott lobe, we observe shrinking in the domain of the first-order phase transition. In particular, for $U_{12}=0.8 U$ and $\rho=2$ Mott lobe, the first-order MI-SF phase transition is observed for $1.1 \lesssim \mu / U \lesssim 1.3$ with the CGMF calculation. While with SGMF, it is $0.99 \lesssim \mu / U \lesssim 1$.44. Similar trends were reported in the comparison of the mean-field theory and quantum Monte Carlo results in Ref. [92]. 


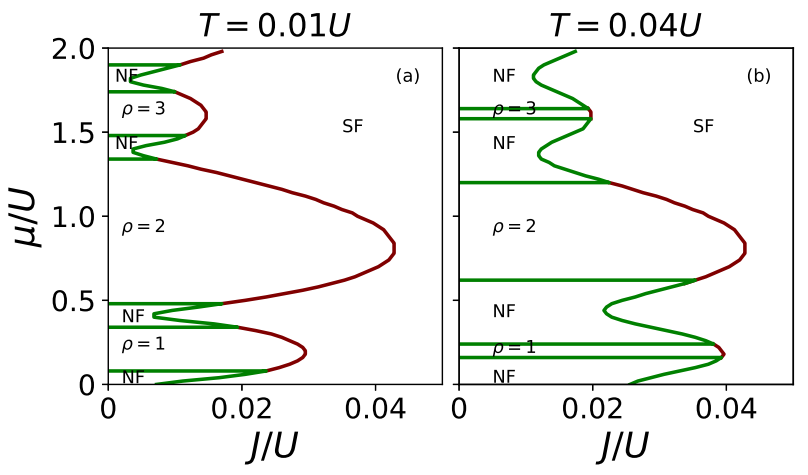

FIG. 2. Phase diagram of TBHM at different temperatures for $U_{12}=$ $0.4 U$. Maroon colored line represents the phase boundaries between the incompressible lobes and the superfluid phase, while the green line forms the boundary of region of the normal fluid (NF) phase.

\section{B. Phase diagram at finite temperatures}

The results we have discussed are at zero temperature, a theoretical simplification. This simplification helps to explore the basic qualitative features of the quantum phases in the system. In these results the thermal fluctuations are absent. Experiments are, however, at finite temperatures and effects of thermal fluctuations have to be incorporated. The competition between the quantum and thermal fluctuations modify the zero-temperature phase diagram. At finite temperatures, the observables have to be calculated with the thermal averaging, and this requires calculation of the partition function. In the mean-field theory we have used, the single-site partition function

$$
Z_{p, q}=\sum_{l} e^{-\beta E_{p, q}^{l}}
$$

where $\beta=1 / k_{B} T, T$ is the temperature of the system, and $E_{p, q}^{l}$ is the $l$ th eigenenergy of the single site Hamiltonian at the lattice site $(p, q)$. As the parameters in the Hamiltonian in Eq.(2) are scaled with the onsite interaction $U$, the temperature of the system is in the units of $U / k_{B}$. And, for simplicity, we set $k_{B}=1$. The thermal average of the superfluid order parameter for the $k$ th species at the $(p, q)$ lattice site is

$$
\left\langle\phi_{p, q}^{k}\right\rangle=\frac{1}{Z_{p, q}} \sum_{l}{ }_{p, q}^{l}\left\langle\psi\left|\hat{b}_{p, q}^{k} e^{-\beta E_{p, q}^{l}}\right| \psi\right\rangle_{p, q}^{l}
$$

where $\langle\ldots\rangle$ represents the thermal averaging and $|\psi\rangle_{p, q}^{l}$ is the $l$ th eigenstate of the single site Hamiltonian. Similarly, the occupancy or the density at finite $T$ is defined as

$$
\left\langle\left\langle\hat{n}_{p, q}^{k}\right\rangle\right\rangle=\frac{1}{Z_{p, q}} \sum_{l}{ }_{p, q}^{l}\left\langle\psi\left|\hat{n}_{p, q}^{k} e^{-\beta E_{p, q}^{l}}\right| \psi\right\rangle_{p, q}^{l} .
$$

For a detail implementation of the finite temperature Gutzwiller method, we refer to Refs. [85, 86]. At finite temperatures, there is an additional phase, normal fluid phase, in the phase diagram. It emerges due to the thermal fluctuations $[94,95]$. This phase has superfluid order parameter $\phi=0$, and the local density is real. To distinguish the normal fluid phase from the incompressible $\rho=n$ lobes, we compute the local compressibility $\kappa$, which is proportional to the local number variance. The $\kappa$ is zero in the incompressible phase, while it is finite for the normal fluid phase. As an example, in the Fig. 2, we show the phase diagrams of the TBHM at $T=0.01 U$ and $0.04 U$ with the same interaction parameters as in Fig.1(b). That is with the interspecies interaction $U_{12}=0.4 U$. In the phase diagrams, the thermal-fluctuationsinduced melting of the incompressible lobes into normal fluid phase is visible. At finite temperature, the normal fluid phase occupies the regions with $\mu$ below and above the tip of the lobes. The domain of this phase is enhanced as the temperature is increased and this is evident from the Fig. 2. This results in the shrinking of the incompressible lobes. Upon increasing the temperature further, the incompressible phases disappear above a critical temperature. For the parameters considered, $T \approx 0.061 U$ is the critical temperature at which the incompressible lobes completely melt.

\section{PHASE DIAGRAM WITH LONG-RANGE INTERACTIONS}

$$
\text { A. } V_{12}=0
$$

The ground state of the eTBHM Hamiltonian in Eq. (8), like in the previous case, is by obtained using the Gutzwiller ansatz. The long-range interactions in the eTBHM introduce two more phases, density wave and supersolid, in the phase diagram. To analyze and highlight the effect of long-range intra- and interspecies interactions, we first consider the case of $V_{12}=0$. And we set the intraspecies $\mathrm{NN}$ interaction strength $V_{k}=0.05 \mathrm{U}$. Then, we vary the interspecies onsite interaction strength $U_{12}$, which can be achieved in experiments through the Feshbach resonance. The choice of low value of $V_{k}$ is based on the parameters realized in dipolar Bose-Einstein Condensate experiments [6]. In these experiments, $V / \hbar$ is in the range $\approx 10-100 \mathrm{~Hz}$, whereas $U / \hbar$ has typical values in $\mathrm{kHz}$. In addition, this choice of parameters has the unique possibility to study the MI-DW quantum phase transition by changing $U_{12}$ and keeping $V_{k}$ fixed. This is to be contrasted with the extended Bose-Hubbard model, where the NN interaction strength $V \geq 0.25 U[73,86]$ marks the critical point for such quantum phase transitions. Like in the case of the Bose-Hubbard model, we consider symmetric hopping $J_{x}^{k}=J_{y}^{k}=J$, identical chemical potential $\tilde{\mu}_{p, q}^{k}=\mu$, and $U_{k k}=U$. The phase diagram for $U_{12}=0$ is shown in Fig. 3 (a). It is identical to the phase diagram of the single species extended Bose-Hubbard model $[73,86]$ and consists of the $\mathrm{DW}(1,0), \mathrm{MI}(1,1), \mathrm{DW}(2,1), \mathrm{MI}(2,2)$, supersolid (green line) and superfluid phases. In the figure, the supersolid phase occurs as a thin envelope around the density wave lobes. On increasing $V_{k}$ but keeping the other parameters fixed, the size of the density wave lobes and the accompanying envelope of the supersolid phase are enhanced. However, the Mott lobes disappear from the phase diagram. This is due to the higher energy cost of having commensurate occupancy due to the in- 


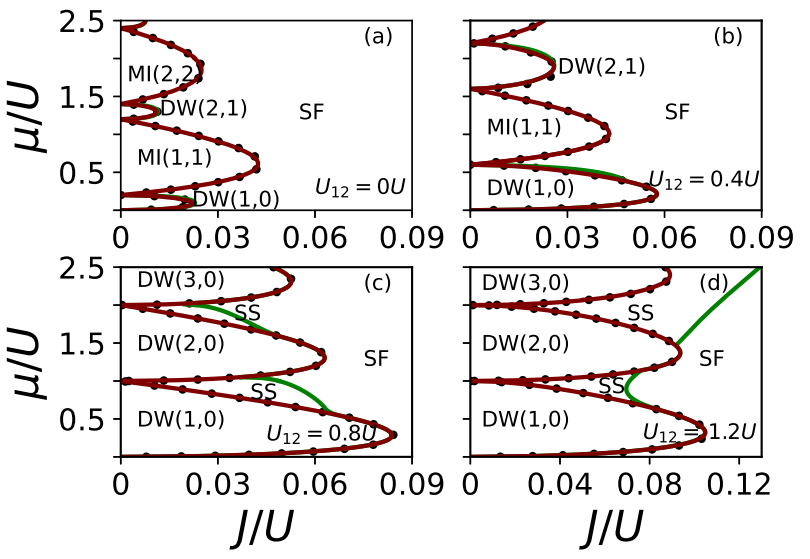

FIG. 3. Phase diagram of eTBHM at different interspecies interaction strength $U_{12}$ and for interspecies NN interaction $V_{12}=V_{21}=$ $0, V_{1}=V_{2}=0.05 \mathrm{U}$. Maroon-colored line forms the boundary of the region comprised of incompressible phases (MI, DW). The filled black dots mark the phase boundaries obtained analytically by perturbative analysis of the mean field Hamiltonian. Around the density wave phase, a supersolid phase exists and the boundary between supersolid and superfluid phases is represented by green lines. The supersolid region around the density wave region enlarges with increasing $U_{12}$. In DW $(n, 0)$ phase both species have $\mathrm{DW}(\mathrm{n}, 0)$ pattern and in $\mathrm{MI}(1,1)$ phase both species have uniform unit occupancy.

traspecies NN interaction. The same effect is reported in the single species extended Bose-Hubbard model $[73,86]$.

The density wave phases with $U_{12}=0$ are fourfold degenerate. Two of the states have $\Delta \rho^{1}=\Delta \rho^{2}$ and the other two have $\Delta \rho^{1}=-\Delta \rho^{2}$. For both set of states, one of the degenerate states is obtained by shifting both of the species by one lattice constant either along the $x$ or $y$ direction. For the $\Delta \rho^{1}=\Delta \rho^{2}$ states, the occupancies of the two species at each lattice sites are the same $n_{p, q}^{1}=n_{p, q}^{2}$. From this the $\Delta \rho^{1}=-\Delta \rho^{2}$ states are obtained after translation of one of the species by one lattice constant either along the $x$ or $y$ direction. Thus, in the latter we have $n^{1, A}=n^{2, B}$ and $n^{1, B}=$ $n^{2, A}$. It is to be noted that the $\rho=1$ phase of the TBHM has the same average density as the DW $(1,0)$. However, the two have different symmetries. The $\rho=1$ phase of the TBHM has atoms from the two species with random occupancies and has no diagonal long-range order. But, the $\mathrm{DW}(1,0)$ has diagonal order arising from the nonzero $\Delta \rho^{k}$. As an example, consider the DW $(1,0)$ phase, the two degenerate states correspond to $\Delta \rho^{1}=\Delta \rho^{2}=1$ and $\Delta \rho^{1}=-\Delta \rho^{2}=1$. At higher $\mu$, the $\operatorname{DW}(2,1)$ intervenes the transition from $\operatorname{MI}(1,1)$ to $\operatorname{MI}(2,2)$ phase.

To study the effect of the interspecies interaction we increase $U_{12}$, retaining $V_{12}$ and $V_{k}$ fixed at 0 and $0.05 U$, respectively. The phase diagram corresponding to $U_{12}=0.4 U$ is shown in Fig. 3 (b). At finite $U_{12}$ the Mott insulator phase is energetically costly due to repulsion between atoms of the two-species coexisting on the same lattice site. So it shifts to higher $\mu / U$ values with increasing $U_{12}$ which can be understood from Eq. (15). As seen from the figure, the finite $U_{12}$ en- hances the $\mathrm{DW}(1,0)$ lobe. The finite $U_{12}$ also lifts the degeneracy of the density wave states, and the state with $n_{p, q}^{1}=n_{p, q}^{2}$ has higher energy. So, the density of the density wave states with finite $U_{12}$ has $n^{1, A}=n^{2, B}$ and $n^{1, B}=n^{2, A}$.

The MI $(1,1)$ lobe remains unchanged in size but is shifted upward in the phase diagram. The shift is attributed to the increase in effective chemical potential arising from the interaction energy associated with finite $U_{12}$. A similar trend, enhancement of the DW $(1,0)$ lobe, occurs in the case of $U_{12}=0$ upon increasing $V_{k}$. In addition to the Mott insulator phase, the DW $(2,1)$ and similar density wave phases with nonzero $n^{k, A}$ and $n^{k, B}$ are also energetically disfavoured. However, the most important feature is the emergence of prominent supersolid phase envelope around each of the density wave lobes. Upon increasing $U_{12}$ further, as seen from the Figs. 3 (c)-3(d), the Mott lobes are transformed into density wave lobes. And, at higher $U_{12}$, only the $\operatorname{DW}(\mathrm{n}, 0)$ phase, with $n \in \mathbb{N}$, is present in the system. The domain of the supersolid phase also increases. Ultimately, the supersolid envelopes around each of density wave lobes merge into a single large supersolid domain, and this is discernible in these figures.

\section{B. $\quad V_{12}>0$}

One of the phenomena unique to the binary condensate is the phase separation. This provides important insights to understand novel phenomena in nonlinear dynamics, pattern formation, quantum phase transitions in condensed-matter systems, etc. [28, 29, 36-42, 45-51, 96]. Phase separation of binary condensates in the weakly interacting regime, as mentioned earlier, is well studied. This, however, is not the case for the strongly interacting two-species ultracold atoms in optical lattices. As discussed earlier, in the TBHM we observe phase separation in the superfluid phase, where the density of the two species are spatially separated into two domains. The phase-separated Mott insulator phases, on the other hand, have random filling of the two species and are not separated into two distinct domains. The inclusion of the NN interactions modifies its density distribution in the phase separated domain. To study this, we solve the Eq. (8) with finite $V_{12}$ and keep it fixed to a value of $0.05 \mathrm{U}$. We, then, increase the interspecies interaction $U_{12}$ from the miscible domain $U_{12}^{2}<U_{11} U_{22}$ to the immiscible domain $U_{12}^{2}>U_{11} U_{22}$. The phase diagrams for selected values of $U_{12}$ are shown in the Fig. 4.

\section{Miscible phase}

In the miscible domain, $U_{12}^{2}<U_{11} U_{22}$, the phase diagram has lobes of incompressible quantum phases having $\rho=n$ with $n \in \mathbb{N}$. These lobes are similar to those in the TBHM. In the present case, however, the $\rho=n$ lobes are intervened by lobes of density wave quantum phases with half-integer total average occupancies $\rho=(2 m+1) / 2$ with $m \in\{0, \mathbb{N}\}$. The total occupancy $n_{p, q}=n_{p, q}^{1}+n_{p, q}^{2}$ of these phases have diagonal long-range order. This is essentially induced by the 


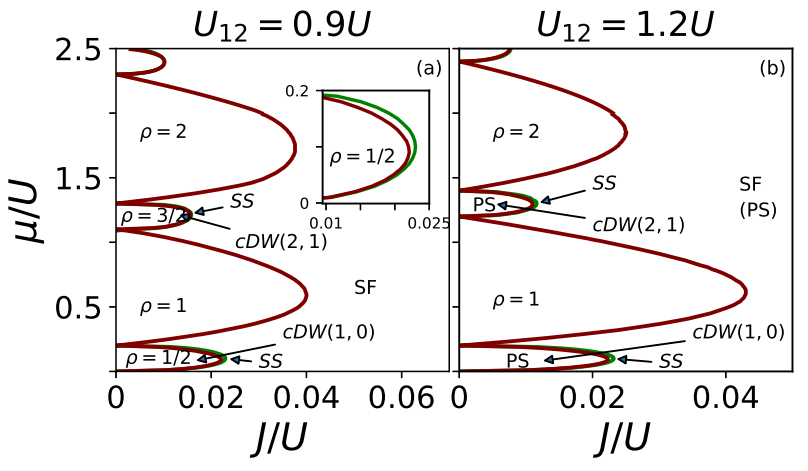

FIG. 4. Phase diagram of eTBHM at the different interspecies interaction strength $U_{12}$ and for interspecies NN interaction $V_{12}=V_{21}=$ $0.05, V_{1}=V_{2}=0.05 \mathrm{U}$. The incompressible (MI, cDW) and compressible phase (SS, SF) regions are separated by maroon colored lines. In correlated density wave phase the two species occupy lattice sites randomly in such a way such that total density $\rho=\rho^{1}+\rho^{2}$ have density wave pattern. And, around this phase, the supersolid phase exists and its boundary is marked by green lines. For $U_{12}=1.2$ the density wave and superfluid phases are phase separated.

nonzero interspecies $\mathrm{NN}$ interaction, $V_{12}>0$. The particle densities $n_{p, q}^{k}$, however, possess no diagonal long-range order. For this reason we refer to these as correlated density wave (cDW) phases. This is to distinguish between the density wave phases with $V_{12}=0$, in which case $n_{p, q}^{k}$ have diagonal long-range order. Due to the small value of the NN interaction strength, the correlated density wave lobes are surrounded by a thin envelope of the supersolid phase. As an example, the phase diagram for $U_{12}=0.9 U$ is shown in Fig. 4(a). In the figure, the $\operatorname{cDW}(1,0)$ has the lowest average occupancy $\rho=1 / 2$. One of the possible density distributions of this phase is $n^{k, A}=0$. And, at the other sublattice the occupancy is $n_{p, q}^{2, B}=1-n_{p, q}^{1, B}$. The values of $n_{p, q}^{1, B}$ are either 0 or 1 , distributed randomly. And, the random distribution implies that there is no diagonal long-range order. In other words, the lattice occupancies of the individual species are not structured but the total lattice occupancy is a structured quantum phase. Around the correlated density wave phase, as $J / U$ is increased for fixed $\mu / U$, the quantum fluctuations drive a second-order quantum phase transition from correlated density wave to the supersolid phase. For the supersolid phase around the $\operatorname{cDW}(1,0)$ phase, the occupancies of the two sublattices are identical, and lie in the range $0 \lesssim n_{p, q}^{1, A}=n_{p, q}^{2, A} \lesssim 0.25$ and $0.25 \lesssim n_{p, q}^{1, B}=n_{p, q}^{2, B} \lesssim 0.50$. Hence, both the species have the same diagonal long-range orders. Here, the occupancies are defined over a finite range due to its finite compressibility. The superfluid order parameters, although different in value, follow similar trends $\phi_{p, q}^{1, A}=\phi_{p, q}^{2, A}$, $\phi_{p, q}^{1, B}=\phi_{p, q}^{2, B}$ and $\phi_{p, q}^{k, B} \neq \phi_{p, q}^{k, A}$. In short, the fluctuations drive the $\mathrm{cDW}(1,0)$ phase with random integer $n_{p, q}^{k, B}$ to identical occupancies. And, $n_{p, q}^{k, A}$ also acquire nonzero values. Upon increasing $J / U$ further, the quantum fluctuations drive another phase transition from the supersolid to the superfluid phase. In this transition, the diagonal long-range order is destroyed and translational invariance of the system is restored.

The insulating phase with average occupancy $\rho=1$, has uniform total lattice occupancy $n_{p, q}=n_{p, q}^{1}+n_{p, q}^{2}=1$. And, the occupancies of the two species satisfy the condition $n_{p, q}^{1}=1-n_{p, q}^{2}$ with $n_{p, q}^{2} \in\{0,1\}$, where the values between the two possibilities are chosen at random. Thus, this phase is like the conventional Mott insulator phase with integer commensurate integer occupancies, but in terms of the total occupancy $n_{p, q}$. Similar to the correlated density wave phase, we refer to this phase as the correlated Mott insulator phase. This implies that increasing the chemical potential or adding more particles to the system, at a fixed but low $J / U$, the system starting from $\mathrm{cDW}(1,0)$ passes through supersolid, superfluid and then to the $\rho=1$ phase. At still higher $\mu$, the $\operatorname{cDW}(2,1)$ phase appears. The total occupancies of the two sublattices in this quantum phase are $n_{p, q}^{A}=n_{p, q}^{1, A}+n_{p, q}^{2, A}=2$ and $n_{p, q}^{B}=n_{p, q}^{1, B}+n_{p, q}^{2, B}=1$. This implies that both species have the same occupancies in the $A$ sublattice $n_{p, q}^{1, A}=n_{p, q}^{2, A}=1$. And, it is equivalent to the $\operatorname{DW}(2,0)$ phase in the eTBHM with $V_{12}=0$. From this phase we obtain the $\operatorname{cDW}(2,1)$ phase by randomly adding one atom of either species at the $B$ sublattice sites. That is, $n_{p, q}^{1, B}=1-n_{p, q}^{2, B}$ with $n_{p, q}^{2, B} \in\{0,1\}$, where the values between the two possibilities are chosen at random. So, effectively, the $\operatorname{cDW}(2,1)$ is a superposition of $\operatorname{DW}(2,0)$ with $\operatorname{cDW}(1,0)$. At higher $\mu$ the other lobes with increasing $\rho$ appear. And these have similar occupancies and order parameter structure as the lobes with lower $\rho$. It is to be highlighted that the phase diagrams are different, qualitatively and quantitatively, from the two-species Bose-Hubbard model where only one of the species is dipolar [79].

The effect of quantum fluctuations are underestimated in the single-site mean-field theory. And this could lead to the appearance of quantum phases which are rendered unstable by quantum fluctuations. The supersolid quantum phase, with diagonal long-range order, is one such phase. So, to check the robustness of the thin supersolid phase around the correlated density wave phase, we use the CGMF theory, with which we study the ground state quantum phases by tiling the system with $2 \times 2$ clusters. With this method, we observe an enhancement of the incompressible lobes. And the extent of the supersolid phase around the $\mathrm{cDW}(1,0)$ phase is similar in size. We also observe the enhancement of $\mathrm{cDW}(1,0)$ lobe along the $\mu / U$ axis. That is, the $\mathrm{cDW}(1,0)$ lobe closes at $\mu=0.3 U$ with CGMF, as compared with $\mu=0.2 U$ calculated by using SGMF. Thus, the supersolid quantum phase around the correlated density wave phases appears to be robust against quantum fluctuations. A concrete observation could be made with larger clusters and by doing a detailed study with cluster finite-size analysis. We shall take this up in our future works.

\section{Immiscible phase}

The criterion for phase separation of the two species in the binary condensates or weakly interacting domain is $U_{12}^{2}>$ $U_{11} U_{22}[34,35]$. And, as discussed earlier, at phase separation the atoms of different species do not occupy the same lattice site. This is the energetically favorable configuration. How- 


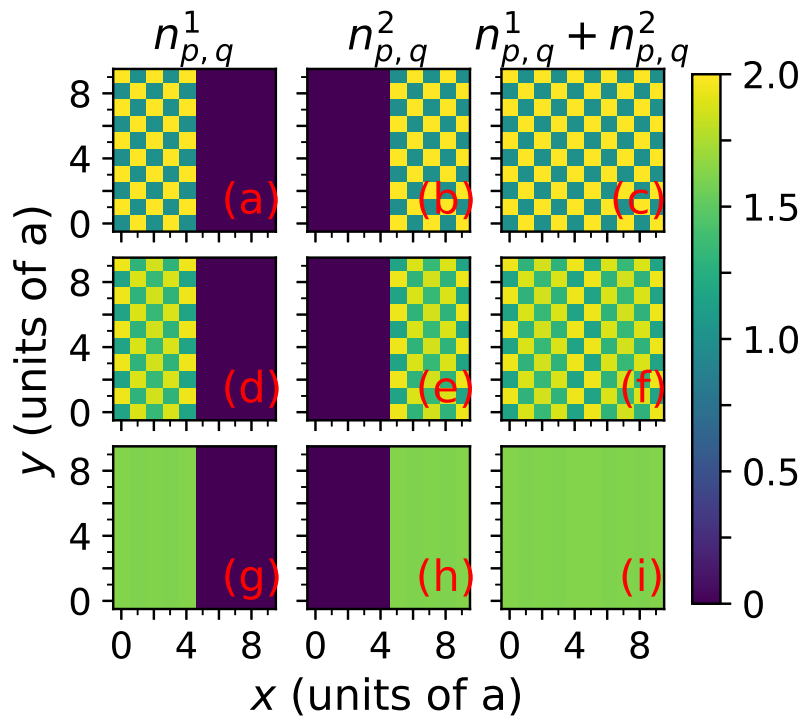

FIG. 5. Phase separation with a side-by-side pattern of species occupancies, obtained with periodic boundary conditions along both the $x$ and $y$ axes. The density distribution of the species over lattice sites is shown in panels (a)-(c) for the $\operatorname{cDW}(2,1)$ phase, in panels (d)-(f) for the supersolid phase, and in panels (g)-(i) for the superfluid phase.

ever, the local nature of the interparticle interaction preserves the inversion symmetry and the species do not separate into two spatial domains. In the binary condensates or weakly interacting domain, the contact interaction is sufficient to break the inversion symmetry and leads to the formation of two spatial domains $[10,12,13,23,24]$ at phase separation. The introduction of the long-range interspecies interaction $\left(V_{12}>0\right)$ in the eTBHM introduces the possibility to lower the energy of the density configurations which breaks inversion symmetry. Thus, there is phase ordering of the two species.

In the present case, for the parameters considered $\left(U_{11}=\right.$ $\left.U_{22}=U\right)$, the phase separation criterion is equivalent to $U_{12}>U$. This choice of parameters, as a representative case, capture the key qualitative and quantitative features of the eTBHM. More importantly, the long-range nature of $V_{12}$ introduces phase ordering at phase separation. As an example, the phase diagram for $U_{12}=1.2 U$ is shown in Fig. 4(b). The structure of the insulating or incompressible and compressible phases are similar to the case of $U_{12}<U$, as shown in Fig. 4(a). But, there is one key difference, the correlated density wave, supersolid and superfluid phases in Fig. 4(b) are phase separated. This is the combined effect of the onsite and long-range interspecies interactions. And, this is indicated in the phase diagram with the annotation PS (phase separated). But the insulating phases with $\rho=1$ and $\rho=2$ are not phase separated. In the $\rho=1$ phase, like in the case of $U_{12}<U$, each lattice site is singly occupied by an atom from the two species chosen randomly. If the phase separation is along one of the axes, say the $x$-axis, the $\operatorname{DW}\left(n_{A}, n_{B}\right)$ phase has occu-

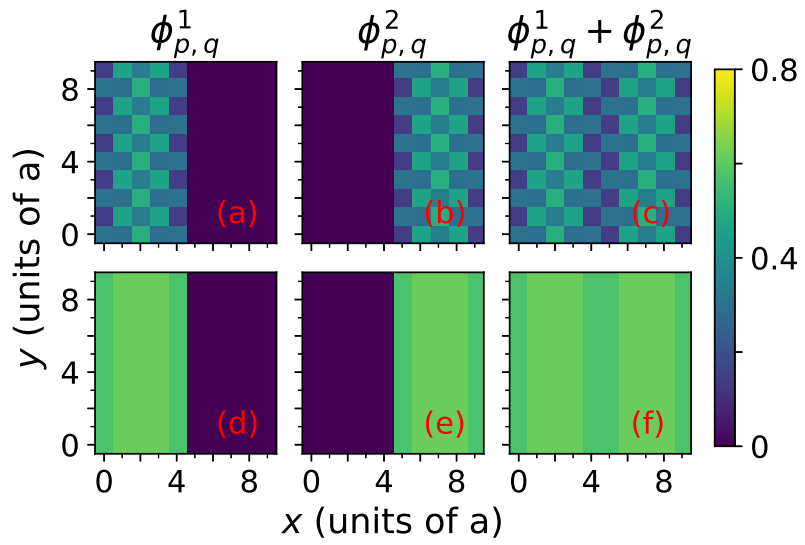

FIG. 6. Phase separation with side-by-side pattern of species superfluid order parameter, obtained with periodic boundary conditions along both $x$ and $y$ axes. The superfluid order parameter at the lattice sites is shown in panels (a)-(c) for supersolid phase and in panels (d)-(f) for the superfluid phase.

pancies

$$
n_{p, q}^{k}= \begin{cases}\Theta\left[(-1)^{k}(p-(K-1) / 2)\right] n_{A} & \text { for }(p, q) \in \mathrm{A} \\ \Theta\left[(-1)^{k}(p-(K-1) / 2)\right] n_{B} & \text { for }(p, q) \in \mathrm{B},\end{cases}
$$

where $k$, as defined earlier, is the species index, $K$ is the size of the system along the $x$-axis, and $n_{A}$ and $n_{B}$ are integers with $n_{A} \neq n_{B}$. The ground state is doubly degenerate because the above density configuration has the same energy when the species are interchanged. The occupancies of other phase-separated phases can also be defined in a similar way. However, in these two phases $n_{A}$ and $n_{B}$ are real. Furthermore, in the supersolid phase $n_{A} \neq n_{B}$ but in the superfluid phase $n_{A}=n_{B}$. The superfluid order parameters for these phases are also defined in the same form. The presence of the Heaviside step functions in Eq. (24) indicates inversion symmetry is broken. The Hamiltonian is, however, invariant under the inversion symmetry. Thus, the phase mixed to separation transition breaks the inversion symmetry spontaneously. And the observed ground state is one of the degenerate configurations.

As an example, the phase diagram in the immiscible domain $U_{12}=1.2 U$ is shown in Fig. 4 (b). In the phase diagram, the global features of the phase domains are qualitatively similar to the phase diagram in the miscible parameter domain $U_{12}=0.9 U$ shown in Fig. 4 (a). There is, however, an important difference. All the phases in the figure are phase separated and this is indicated in the phase diagram with label PS. In the superfluid phase, phase separation occurs across the whole parameter domain. The density profiles of the $\operatorname{cDW}(2,1)$ phase, and the supersolid and superfluid phases around it are shown in Fig.(5). In the figure, consider the lattice sites with odd (even) values of $(p+q)$ as the $A(B)$ sublattice. And, for better representation of the density orders of the structured phases, we consider a system size of $10 \times 10$. Then, from the density pattern in Figs.(5)(a) and 5(b), both the species have 
occupancies $n_{A}=2$ and $n_{B}=1$. And, because it is phase separated, from Eq. (24),

$$
n_{p, q}^{k}= \begin{cases}\Theta\left[(-1)^{k}\left(p-\frac{9}{2}\right)\right] 2 & \text { for odd }(p+q) \\ \Theta\left[(-1)^{k}\left(p-\frac{9}{2}\right)\right] 1 & \text { for even }(p+q) .\end{cases}
$$

The density pattern shown in the figures Figs.(5)(a)-5(c) correspond to the parameters $\mu / U=1.35$ and $J / U=0.010$. The above occupancies of the species imply that each of the species are confined within a subsystem of a $5 \times 10$ lattice. The other species, because we apply periodic boundary conditions along both directions, effectively provides a confining potential. This is better visualized when the system is mapped to a torus. Then, phase separation along one of the axes, divides the torus into two equal halves. Here each half is occupied by one of the species. For such a configuration, there are two interspecies boundaries which segregate the two species. Thus, with a $10 \times 10$ system size, the total length of the boundary is $20 a$, where $a$ (as defined earlier) is the lattice constant. From the figure it is evident that other configuration is the phaseseparated state, existing along the diagonal. This, however, is energetically not favourable as it has larger interface energy due to longer boundary $10(2+\sqrt{2}) a$.

For the same value of chemical potential $\mu / U=1.35$, on increasing the hopping amplitude to $J / U=0.011$ we are in the supersolid phase domain. It is also phase separated and the lattice site occupancies have a similar form as Eq.(25). The occupancies are real, have checkerboard order, and are shown in Fig.(5)(d)-5(f). Another important point is, as seen from the figures, that boundary effects are present in the superfluid order parameter. The reason is that the effective potential which segregates the two species is like a soft boundary condition. And, this is due to the long-range interspecies interaction. The supersolid phase is a superfluid phase with diagonal long-range order, and hence has a nonzero superfluid order parameter $\phi_{p, q}^{k}$. The superfluid order parameters of the two species are shown in Fig.6(a)-6(c). The boundary effects are more prominent in these figures and at the boundaries, the deviations from the checkerboard order of $\phi_{p, q}^{k}$ are visible without ambiguity. It is to be mentioned here that the domain of the supersolid phase, for the parameters considered, is rather small. Despite this, supersolid quantum phase with phase segregation is a novel one and it deserves detailed investigations. Upon increasing $J / U$ further, we reach the superfluid phase, which is also phase separated. As an example, the occupancies and superfluid order parameters for $\mu / U=1.35$ and $J / U=0.015$ are shown in Figs.(5)(g)-5(i) and in Figs.6(d)6(f), respectively. In the superfluid phase, there is phase separation, but the occupancies and superfluid order parameter are uniform within the domains of each species. Thus, the average occupancies and lattice site occupancies are the same $n_{p, q}^{1}=\rho^{1}$ (for $p<5$ ) and $n_{p, q}^{2}=\rho^{2}$ (for $p \geqslant 5$ ). As we consider identical parameters for both the species $\rho=\rho^{1}=\rho^{2}$, where $\rho \in \mathbb{R}$ and $1 \leqslant \rho \leqslant 2$. The values and range are also discernible from the figures. The key point from these case studies is that, for nonzero interspecies long-range interactions and $U_{12}^{2}>U_{11} U_{22}$, the eTBHM has quantum phases which are phase separated.

\section{Finite tilt angle and finite temperature}

The results of the eTBHM discussed so far are the quantum phases of the model described by the Hamiltonian in Eq.7. As mentioned earlier, this corresponds to the case of dipoles aligned perpendicular to the lattice plane. In this section we provide a brief discussion on the general case, where the tilt angle $\theta$ is nonzero. For this, we consider the dipole-dipole interaction

$$
\frac{C_{\mathrm{dd}}}{2} \sum_{i j} \hat{n}_{i} \hat{n}_{j} \frac{\left(1-3 \cos ^{2} \alpha_{i j}\right)}{\left|\mathbf{r}_{i}-\mathbf{r}_{j}\right|^{3}}
$$

where the angle $\alpha_{i j}$ is the angle between the dipole polarization axis and the separation vector $\mathbf{r}_{i}-\mathbf{r}_{j}$ between the lattice sites $i$ and $j$. The coupling constant $C_{\mathrm{dd}}$ represents the strength of the dipole interaction. Without loss of generality, the dipoles are assumed to be polarized in the $y-z$ plane, and then, $\theta=\frac{\pi}{2}-\alpha_{i j}$. The detailed physical description of such a system is given in Ref. [7]. Even though the dipoledipole interaction is a long-range interaction, we restrict it to the NN sites. This simplified limit is sufficient to examine the effects arising from the anisotropy of the interaction. The strength of the dipole-dipole interaction can be varied from $C_{\mathrm{dd}}$ to $-2 C_{\mathrm{dd}}$, by changing $\theta$ from 0 to $\frac{\pi}{2}$. Thus the effective dipole-dipole interaction strength decreases as $\theta$ increases. In the repulsive domain, the decrease in the effective interaction strength shrinks the density wave and Mott lobes. We have verified the decrease of the density wave lobes by considering the tilt angle $\theta=\frac{\pi}{12}$. In the miscible phase $\left(U_{12}=0.9 U\right)$, the phase diagram at $\theta=\frac{\pi}{12}$ is qualitatively similar, but there are quantitative differences in terms of the phase boundaries of the incompressible phases. As stated earlier, we observe that the correlated density wave lobes shrink along the $J / U$ axis and close at a smaller $\mu / U$ value. The thin envelope of the supersolid phase also show the same trends as the correlated density wave lobes. That is, the supersolid phase also shrinks along the $J / U$ axis, and closes at a lesser $\mu / U$ value. The incompressible $\rho=n$ lobes are shifted downward along the $\mu$ axis.

Earlier, we had discussed the ground-state phases with thermal fluctuations associated with finite temperatures. We, similarly, have studied the effects of the thermal fluctuations on the ground-state phases of eTBHM, in particular, for the parameters domain where the system is in the miscible domain. We observe that the regions of the incompressible lobes are reduced, and the melted region is occupied by the normal fluid phase. Like in the case of TBHM, in Sec. III B, an increase in temperature shrinks the incompressible lobes. And, above a critical temperature, the lobes disappear.

\section{Linear Stability analysis}

The dynamics of fluid mixtures exhibit different types of instabilities. The binary condensates are no exception. In particular, the Rayleigh-Taylor instability [27, 28] and KelvinHelmholtz instability [97, 98] have been studied in detail. 
So, it is pertinent to examine the stability of the spatially phase-separated ground-state configuration of the eTBHM. The collective excitations are the relevant properties of the system which carry signatures of instabilities. To calculate the collective excitations we add fluctuations $\delta c_{n_{1}, n_{2}}^{(p, q)}(t)$ to the ground-state coefficients in the dynamical Gutzwiller meanfield equation $[99,100]$. The coefficients of the Fock states in Eq. 4 is then modified to

$$
c_{n_{1}, n_{2}}^{(p, q)}(t)=\bar{c}_{n_{1}, n_{2}}^{(p, q)}+\delta c_{n_{1}, n_{2}}^{(p, q)}(t)
$$

where $\bar{c}_{n_{1}, n_{2}}^{(p, q)}$ are the coefficients at equilibrium or the groundstate solution of the Gutzwiller mean-field theory. To obtain the collective excitations, we use the Bogoliubov approximation and define

$$
\delta c_{n_{1}, n_{2}}^{(p, q)}(t)=u_{n_{1}, n_{2}}^{(p, q)} e^{-i \omega t}+v_{n_{1}, n_{2}}^{*(p, q)} e^{i \omega t},
$$

where $\omega$ is the energy of the collective mode, and $\left(u_{n_{1}, n_{2}}, v_{n_{1}, n_{2}}\right)$ is the amplitude of the collective modes [101-103]. Using this in the dynamical Gutzwiller equation, and retaining terms linear in $u$ and $v$, we obtain the Bogoliubov-de Gennes equation. The details of the derivation and equations for the eTBHM are given in Appendix C. We, then, diagonalize the Bogoliubov-de Gennes matrix and obtain the eigenspectrum of the system.

In the eigenspectrum of the system, the appearance of collective modes with complex energies is a signature of dynamical instability. With complex energy, the imaginary part leads to an exponential growth of the fluctuations and this is evident from Eq.(27). And, thus, the system is unstable to perturbations. To determine the stability of the phases in the phase diagram presented in Fig. 4 (b), we have performed the stability analysis for the phase-separated, side-by-side ordered cDW $(2,1)$ and superfluid phases. In both of these phases, we get a real-valued excitation spectrum. This indicates that these phase-separated states are dynamically stable. We have also verified the stability of other phases in the phase diagram.

\section{CONCLUSIONS}

In conclusion, we obtain the phase diagram of the twospecies Bose-Hubbard model and its extended version, the eTBHM with long-range interactions in two-dimensional optical lattices. Our findings are pertinent and timely in view of the recent experimental realization of the Er-Dy binary dipolar Bose-Einstein condensate mixture [62]. The phase diagram of the TBHM has the unique feature of additional Mott lobes with average occupancies which are half integer. These lobes emerge due to the presence of the second species. And, the domain of these lobes are enhanced with the increase of the interspecies interaction strength. In the case of eTBHM, we obtain insulating phases with the nonoverlapping density distributions even with $U_{12}^{2}<U_{11} U_{22}$, where the atoms of the two species are distributed across the system randomly. The nonoverlapping densities are like phase separation but, in this work, we use phase separation to mean the configuration where the densities of the two species are segregated into two nonoverlapping domains. One key finding of our study is that the DW-MI quantum phase transitions may occur by varying $U_{12}$ while keeping $V_{k}$ fixed. This is in contrast with the single species extended Bose-Hubbard model, where the $\mathrm{NN}$ interaction strength is required to be large to observe such quantum phase transitions. With finite interspecies $\mathrm{NN}$ interactions, we obtain the phase diagram in the miscible and immiscible regimes. Our result is that the correlated density wave, supersolid, and superfluid phases in the eTBHM in the immiscible domain $U_{12}^{2}>U_{11} U_{22}$ are phase separated. And, they have side by side order. These phase-separated phases are dynamically stable.

\section{ACKNOWLEDGEMENTS}

The results presented in the paper are based on computations using Vikram-100, the 100TFLOP HPC Cluster at the Physical Research Laboratory, Ahmedabad, India. K.S. acknowledges the support of the National Science Centre, Poland via project 2016/21/B/ST2/01086.

\section{Appendix A: Perturbation Analysis of the Two-Species Bose-Hubbard Model}

The unperturbed ground state at the lattice site $(p, q)$ has the form $|\psi\rangle_{p, q}^{(0)}=\left|n^{1}, n^{2}\right\rangle_{p, q}$. The energy of this unperturbed ground state is

$$
\begin{aligned}
E_{n_{p, q}^{1}, n_{p, q}^{2}}^{(0)} & =\frac{U}{2}\left[n_{p, q}^{1}\left(n_{p, q}^{1}-1\right)+n_{p, q}^{2}\left(n_{p, q}^{2}-1\right)\right] \\
& +U_{12} n_{p, q}^{1} n_{p, q}^{2}-\mu_{p, q}^{1} n_{p, q}^{1}-\mu_{p, q}^{2} n_{p, q}^{2}
\end{aligned}
$$

where we have chosen $U_{11}=U_{22}=U$. Then, to the first order of the superfluid order parameter $\phi_{p, q}^{k}$ the perturbed ground state can be written as

$$
\begin{aligned}
|\psi\rangle_{p, q} & =\left|n^{1}, n^{2}\right\rangle_{p, q} \\
& +\sum_{\substack{m^{1}, m^{2} \\
\neq n^{1}, n^{2}}} \frac{p, q\left\langle m^{1}, m^{2}\left|\hat{h}_{p, q, 1}^{\mathrm{TBH}}\right| n^{1}, n^{2}\right\rangle_{p, q}}{E_{n_{p, q}^{1}, n_{p, q}^{2}}^{0}-E_{m_{p, q}^{1}, m_{p, q}^{2}}^{0}}\left|m^{1}, m^{2}\right\rangle_{p, q},
\end{aligned}
$$

where, considering uniform hopping strengths for both the species $\left(J_{x}^{1}=J_{x}^{2}=J_{y}^{1}=J_{y}^{2}=J\right)$ and superfluid order parameters as real numbers

$$
\hat{h}_{p, q, 1}^{\mathrm{TBH}}=-J\left[\bar{\phi}_{p, q}^{1}\left(\hat{b}_{p, q}^{\dagger 1}+\hat{b}_{p, q}^{1}\right)+\bar{\phi}_{p, q}^{2}\left(\hat{b}_{p, q}^{\dagger 2}+\hat{b}_{p, q}^{2}\right)\right]
$$


with $\bar{\phi}_{p, q}^{k}=\left(\phi_{p+1, q}^{k}+\phi_{p-1, q}^{k}+\phi_{p, q+1}^{k}+\phi_{p, q-1}^{k}\right)$. Then, using Eqs. (A1)-(A3) the ground state can be calculated as

$$
\begin{aligned}
|\psi\rangle_{p, q}= & \left|n^{1}, n^{2}\right\rangle_{p, q} \\
& +J \bar{\phi}_{p, q}^{1}\left\{\frac{\sqrt{n_{p, q}^{1}+1}}{n_{p, q}^{1} U-\mu_{p, q}^{1}+U_{12} n_{p, q}^{2}}\left|n^{1}+1, n^{2}\right\rangle_{p, q}\right. \\
& \left.-\frac{\sqrt{n_{p, q}^{1}}}{\left(n_{p, q}^{1}-1\right) U-\mu_{p, q}^{1}+U_{12} n_{p, q}^{2}}\left|n^{1}-1, n^{2}\right\rangle_{p, q}\right\} \\
& +J \bar{\phi}_{p, q}^{2}\left\{\frac{\sqrt{n_{p, q}^{2}+1}}{n_{p, q}^{2} U-\mu_{p, q}^{2}+U_{12} n_{p, q}^{1}}\left|n^{1}, n^{2}+1\right\rangle_{p, q}\right. \\
& -\frac{\sqrt{n_{p, q}^{2}}}{\left(n_{p, q}^{2}-1\right) U-\mu_{p, q}^{2}+U_{12} n_{p, q}^{1}}\left|n^{1}, n^{2}-1\right\rangle_{p, q}
\end{aligned} .
$$

From this state, we calculate the superfluid order parameter $\phi_{p, q}^{k}={ }_{p, q}\left\langle\psi\left|\hat{b}_{p, q}^{k}\right| \psi\right\rangle_{p, q}$, and the expression is given in Eq. (11).

\section{Appendix B: Perturbation analysis of the Extended Two-Species Bose-Hubbard Model}

The unperturbed ground state at the lattice site $(p, q) \in A$ sublattice has the form $|\psi\rangle_{A}^{(0)}=\left|n^{1, A}, n^{2, A}\right\rangle$ with energy

$$
\begin{aligned}
E_{n^{1, A}, n^{2, A}}^{(0)} & =\frac{U}{2}\left[n^{1, A}\left(n^{1, A}-1\right)+n^{2, A}\left(n^{2, A}-1\right)\right] \\
& +U_{12} n^{1, A} n^{2, A}-\mu\left(n^{1, A}+n^{2, A}\right) \\
& +4 V_{1}\left(n^{1, A} n^{1, B}+n^{2, A} n^{2, B}\right)
\end{aligned}
$$

where we have chosen $\tilde{\mu}^{1}=\tilde{\mu}^{2}=\mu, U_{11}=U_{22}=U$ and $V_{1}=V_{2}$. Then, to first order in the superfluid order parameter $\phi_{p, q}^{k}$, the ground state is

$$
\begin{gathered}
|\psi\rangle_{A}=\left|n^{1}, n^{2}\right\rangle_{A}+ \\
\sum_{\substack{\left(m^{1}, m^{2}\right) \\
\neq\left(n^{1}, n^{2}\right)}} \frac{A\left\langle m^{1}, m^{2}\left|\hat{h}_{p, q, 1}^{\mathrm{TBH}}\right| n^{1}, n^{2}\right\rangle_{A}}{E_{n^{1, A}, n^{2}, A}^{0}-E_{m^{1, A}, m^{2, A}}^{0}}\left|m^{1}, m^{2}\right\rangle_{A}
\end{gathered}
$$

where, considering $J_{x}^{1}=J_{x}^{2}=J_{y}^{1}=J_{y}^{2}=J$ and the superfluid order parameters as real numbers

$$
\hat{h}_{p, q, 1}^{\mathrm{TBH}}=-4 J\left[\phi_{B}^{1}\left(\hat{b}_{A}^{\dagger 1}+\hat{b}_{A}^{1}\right)+\phi_{B}^{2}\left(\hat{b}_{A}^{\dagger 2}+\hat{b}_{A}^{2}\right)\right] .
$$

Then, using Eqs. (B1)-(B3) the perturbed ground state is

$|\psi\rangle_{A}=\left|n^{1, A}, n^{2, A}\right\rangle$

$$
\begin{aligned}
& +4 J \phi_{B}^{1}\left\{\frac{\sqrt{n^{1, A}+1}}{U n^{1, A}-\mu+U_{12} n^{2, A}+4 V_{1} n^{1, B}}\left|n^{1, A}+1, n^{2, A}\right\rangle\right. \\
& \left.-\frac{\sqrt{n^{1, A}}}{U\left(n^{1, A}-1\right)-\mu+U_{12} n^{2, A}+4 V_{1} n^{1, B}}\left|n^{1, A}-1, n^{2, A}\right\rangle\right\} \\
& +4 J \phi_{B}^{2}\left\{\frac{\sqrt{n^{2, A}+1}}{U n^{2, A}-\mu+U_{12} n^{1, A}+4 V_{1} n^{2, B}}\left|n^{1, A}, n^{2, A}+1\right\rangle\right. \\
& \left.-\frac{\sqrt{n^{2, A}}}{U\left(n^{2, A}-1\right)-\mu+U_{12} n^{1, A}+4 V_{1} n^{2, B}}\left|n^{1, A}, n^{2, A}-1\right\rangle\right\} .
\end{aligned}
$$

Using this, the superfluid order parameter $\phi_{A}^{1}={ }_{A}\left\langle\psi\left|\hat{b}_{A}^{1}\right| \psi\right\rangle_{A}$ is given by

$$
\begin{aligned}
\phi_{A}^{1} & =4 J \phi_{B}^{1}\left\{\frac{n^{1, A}+1}{U n^{1, A}-\mu+U_{12} n^{2, A}+4 V_{1} n^{1, B}}\right. \\
& \left.-\frac{n^{1, A}}{U\left(n^{1, A}-1\right)-\mu+U_{12} n^{2, A}+4 V_{1} n^{1, B}}\right\} .
\end{aligned}
$$

A similar analysis can be done at the lattice site $(p, q) \in B$ to obtain the superfluid order parameter $\phi_{B}^{1}={ }_{B}\left\langle\psi\left|\hat{b}_{B}^{1}\right| \psi\right\rangle_{B}$, and we get

$$
\begin{aligned}
\phi_{B}^{1} & =4 J \phi_{A}^{1}\left\{\frac{n^{1, B}+1}{U n^{1, B}-\mu+U_{12} n^{2, B}+4 V_{1} n^{1, A}}\right. \\
& \left.-\frac{n^{1, B}}{U\left(n^{1, B}-1\right)-\mu+U_{12} n^{2, B}+4 V_{1} n^{1, A}}\right\} .
\end{aligned}
$$

Substituting $\phi_{B}^{1}$ from Eq.(B6) into Eq.(B5) and then, taking the limit $\phi_{A}^{1} \rightarrow 0^{+}$gives Eq.(16), which defines the DWcompressible phase boundary.

\section{Appendix C: Bogoliubov-de Gennes equations for eTBHM}

The Bogoliubov-de Gennes equation, obtained after retaining the linear terms in the fluctuations and using Bogoliubov approximation, for the eTBHM is

$$
\begin{gathered}
\omega u_{n_{1}, n_{2}}^{(p, q)}=\sum_{\left(p^{\prime}, q^{\prime}\right), m_{1}, m_{2}}\left(A_{\left(p^{\prime}, q^{\prime}\right) m_{1}, m_{2}}^{(p, q) n_{1}, n_{2}} u_{m_{1}, m_{2}}^{\left(p^{\prime}, q^{\prime}\right)}+B_{\left(p^{\prime}, q^{\prime}\right) m_{1}, m_{2}}^{(p, q) n_{1}, n_{2}} v_{m_{1}, m_{2}}^{\left(p^{\prime}, q^{\prime}\right)}\right), \\
-\omega v_{n_{1}, n_{2}}^{(p, q)}=\sum_{\left(p^{\prime}, q^{\prime}\right), m_{1}, m_{2}}\left(B_{\left(p^{\prime}, q^{\prime}\right) m_{1}, m_{2}}^{*(p, q) n_{1}, n_{2}} u_{m_{1}, m_{2}}^{\left(p^{\prime}, q^{\prime}\right)}+A_{\left(p^{\prime}, q^{\prime}\right) m_{1}, m_{2}}^{*(p, q) n_{1}, n_{2}} v_{m_{1}, m_{2}}^{\left(p^{\prime}, q^{\prime}\right)}\right) .
\end{gathered}
$$

It is a set of two coupled equations in terms of the mode amplitudes $u$ and $v$. The matrix elements in the above equations are 


$$
\begin{aligned}
& A_{\left(p^{\prime}, q^{\prime}\right), m_{1}, m_{2}}^{(p, q), n_{1}, n_{2}}=\left(\left[\sum_{k}\left(\frac{U_{k k}}{2} n_{k}\left(n_{k}-1\right)-\mu^{k} n_{k}+V_{k} n_{k} \mathbf{N}_{(p, q)}^{k}\right)+U_{12} n_{1} n_{2}+V_{12}\left(n_{1} \mathbf{N}_{(p, q)}^{2}+n_{2} \mathbf{N}_{(p, q)}^{1}\right)-\omega_{0}^{(p, q)}\right] \delta_{n_{1}, m_{1}} \delta_{n_{2}, m_{2}}\right. \\
& -J^{1}\left[\sqrt{n_{1}+1} \Phi_{(p, q)}^{1 *} \delta_{m_{1}, n_{1}+1}+\sqrt{n_{1}} \Phi_{(p, q)}^{1} \delta_{m_{1}, n_{1}-1}\right] \delta_{m_{2}, n_{2}} \\
& \left.-J^{2}\left[\sqrt{n_{2}+1} \Phi_{(p, q)}^{2 *} \delta_{m_{2}, n_{2}+1}+\sqrt{n_{2}} \Phi_{(p, q)}^{2} \delta_{m_{2}, n_{2}-1}\right] \delta_{m_{1}, n_{1}}\right) \delta_{p^{\prime}, p} \delta_{q^{\prime}, q} \\
& +\left(-J^{1} \sqrt{\left(n_{1}+1\right)\left(m_{1}+1\right)} \bar{c}_{m_{1}+1, m_{2}}^{*\left(p^{\prime}, q^{\prime}\right)} \bar{c}_{n_{1}+1, n_{2}}^{(p, q)}-J^{1} \sqrt{n_{1} m_{1}} \bar{c}_{m_{1}-1, m_{2}}^{*\left(p^{\prime}, q^{\prime}\right)} \bar{c}_{n_{1}-1, n_{2}}^{(p, q)}\right. \\
& -J^{2} \sqrt{\left(n_{2}+1\right)\left(m_{2}+1\right)} \bar{c}_{m_{1}, m_{2}+1}^{*\left(p^{\prime}, q^{\prime}\right)} \bar{c}_{n_{1}, n_{2}+1}^{(p, q)}-J^{2} \sqrt{n_{2} m_{2}} \bar{c}_{m_{1}, m_{2}-1}^{*\left(p^{\prime}, q^{\prime}\right)} \bar{c}_{n_{1}, n_{2}-1}^{(p, q)} \\
& \left.+\left[V_{1} n_{1} m_{1}+V_{2} n_{2} m_{2}+V_{12}\left(n_{1} m_{2}+n_{2} m_{1}\right)\right] \bar{c}_{m_{1}, m_{2}}^{*\left(p^{\prime}, q^{\prime}\right)} \bar{c}_{n_{1}, n_{2}}^{(p, q)}\right)\left(\delta_{p^{\prime}, p \pm 1} \delta_{q^{\prime}, q}+\delta_{p^{\prime}, p} \delta_{q^{\prime}, q \pm 1}\right), \\
& B_{\left(p^{\prime}, q^{\prime}\right), m_{1}, m_{2}}^{(p, q), n_{1}, n_{2}}=\left(-J^{1} \sqrt{\left(n_{1}+1\right)\left(m_{1}\right)} \bar{c}_{m_{1}-1, m_{2}}^{\left(p^{\prime}, q^{\prime}\right)} \bar{c}_{n_{1}+1, n_{2}}^{(p, q)}-J^{1} \sqrt{n_{1}\left(m_{1}+1\right)} \bar{c}_{m_{1}+1, m_{2}}^{\left(p^{\prime}, q^{\prime}\right)} \bar{c}_{n_{1}-1, n_{2}}^{(p, q)}\right. \\
& -J^{2} \sqrt{\left(n_{2}+1\right)\left(m_{2}\right)} \bar{c}_{m_{1}, m_{2}-1}^{\left(p^{\prime}, q^{\prime}\right)} \bar{c}_{n_{1}, n_{2}+1}^{(p, q)}-J^{2} \sqrt{n_{2}\left(m_{2}+1\right)} \bar{c}_{m_{1}, m_{2}+1}^{\left(p^{\prime}, q^{\prime}\right)} \bar{c}_{n_{1}, n_{2}-1}^{(p, q)} \\
& \left.+\left[V_{1} n_{1} m_{1}+V_{2} n_{2} m_{2}+V_{12}\left(n_{1} m_{2}+n_{2} m_{1}\right)\right] \bar{c}_{m_{1}, m_{2}}^{\left(p^{\prime}, q^{\prime}\right)} \bar{c}_{n_{1}, n_{2}}^{(p, q)}\right)\left(\delta_{p^{\prime}, p \pm 1} \delta_{q^{\prime}, q}+\delta_{p^{\prime}, p} \delta_{q^{\prime}, q \pm 1}\right) .
\end{aligned}
$$

Here $k$ represents the species index. $\Phi_{(p, q)}^{k}$ and $\mathbf{N}_{(p, q)}^{k}$ are the mean-field superfluid order parameter and the number density summed over NN sites of $(p, q)$ respectively. And $\omega_{0}^{(p, q)}$ is the ground-state energy calculated using the unperturbed coefficients. The equations can be written as a matrix equation

$$
\omega\left(\begin{array}{l}
\mathbf{u} \\
\mathbf{v}
\end{array}\right)=\left(\begin{array}{cc}
\mathbf{A} & \mathbf{B} \\
-\mathbf{B}^{*} & -\mathbf{A}^{*}
\end{array}\right)\left(\begin{array}{l}
\mathbf{u} \\
\mathbf{v}
\end{array}\right) .
$$

The matrix on the right-hand side is the Bogoliubov-de Gennes matrix. Diagonalizing, we get the collective modes of the system.
[1] J. Hubbard, "Electron correlations in narrow energy bands," Proc. Royal Soc. A 276, 238 (1963).

[2] M. P. A. Fisher, P. B. Weichman, G. Grinstein, and D. S. Fisher, "Boson localization and the superfluid-insulator transition,” Phys. Rev. B 40, 546 (1989).

[3] D. Jaksch, C. Bruder, J. I. Cirac, C. W. Gardiner, and P. Zoller, "Cold bosonic atoms in optical lattices," Phys. Rev. Lett. 81, 3108 (1998).

[4] M. Greiner, O. Mandel, T. Esslinger, T. W. Hänsch, and I. Bloch, "Quantum phase transition from a superfluid to a Mott insulator in a gas of ultracold atoms," Nature (London) 415, 39 (2002).

[5] Till D. Kühner, Steven R. White, and H. Monien, "Onedimensional bose-hubbard model with nearest-neighbor interaction,” Phys. Rev. B 61, 12474-12489 (2000).

[6] S. Baier, M. J. Mark, D. Petter, K. Aikawa, L. Chomaz, Z. Cai, M. Baranov, P. Zoller, and F. Ferlaino, "Extended bose-hubbard models with ultracold magnetic atoms," Science 352, 201-205 (2016).

[7] Soumik Bandyopadhyay, Rukmani Bai, Sukla Pal, K. Suthar, Rejish Nath, and D. Angom, "Quantum phases of canted dipolar bosons in a two-dimensional square optical lattice," Phys. Rev. A 100, 053623 (2019).

[8] G. Modugno, M. Modugno, F. Riboli, G. Roati, and M. In- guscio, "Two atomic species superfluid," Phys. Rev. Lett. 89, 190404 (2002).

[9] A.D. Lercher, T. Takekoshi, M. Debatin, B. Schuster, R. Rameshan, F. Ferlaino, R. Grimm, and H.-C. Nägerl, "Production of a dual-species Bose-Einstein condensate of $\mathrm{Rb}$ and Cs atoms," Eur. Phys. J. D 65, 3 (2011).

[10] D. J. McCarron, H. W. Cho, D. L. Jenkin, M. P. Köppinger, and S. L. Cornish, "Dual-species Bose-Einstein condensate of ${ }^{87} \mathrm{Rb}$ and ${ }^{133}$ Cs," Phys. Rev. A 84, 011603 (2011).

[11] Benjamin Pasquiou, Alex Bayerle, Slava M. Tzanova, Simon Stellmer, Jacek Szczepkowski, Mark Parigger, Rudolf Grimm, and Florian Schreck, "Quantum degenerate mixtures of strontium and rubidium atoms," Phys. Rev. A 88, 023601 (2013).

[12] L. Wacker, N. B. Jørgensen, D. Birkmose, R. Horchani, W. Ertmer, C. Klempt, N. Winter, J. Sherson, and J. J. Arlt, "Tunable dual-species Bose-Einstein condensates of ${ }^{39} \mathrm{~K}$ and ${ }^{87}$ Rb," Phys. Rev. A 92, 053602 (2015).

[13] Fudong Wang, Xiaoke Li, Dezhi Xiong, and Dajun Wang, “A double species ${ }^{23} \mathrm{Na}$ and ${ }^{87} \mathrm{Rb}$ Bose-Einstein condensate with tunable miscibility via an interspecies Feshbach resonance," J. Phys. B 49, 015302 (2016).

[14] C. J. Myatt, E. A. Burt, R. W. Ghrist, E. A. Cornell, and C. E. Wieman, "Production of two overlapping Bose-Einstein condensates by sympathetic cooling," Phys. Rev. Lett. 78, 586 
(1997).

[15] D. S. Hall, M. R. Matthews, J. R. Ensher, C. E. Wieman, and E. A. Cornell, "Dynamics of component separation in a binary mixture of Bose-Einstein condensates," Phys. Rev. Lett. 81, 1539 (1998).

[16] D. M. Stamper-Kurn, M. R. Andrews, A. P. Chikkatur, S. Inouye, H.-J. Miesner, J. Stenger, and W. Ketterle, "Optical confinement of a Bose-Einstein condensate," Phys. Rev. Lett. 80, 2027 (1998).

[17] J. Stenger, S. Inouye, D. M. Stamper-Kurn, H.-J. Miesner, A. P. Chikkatur, and W. Ketterle, "Spin domains in groundstate Bose-Einstein condensates," Nature (London) 396, 345 (1998).

[18] P. Maddaloni, M. Modugno, C. Fort, F. Minardi, and M. Inguscio, "Collective oscillations of two colliding Bose-Einstein condensates," Phys. Rev. Lett. 85, 2413 (2000).

[19] G. Delannoy, S. G. Murdoch, V. Boyer, V. Josse, P. Bouyer, and A. Aspect, "Understanding the production of dual BoseEinstein condensation with sympathetic cooling," Phys. Rev. A 63, 051602 (2001).

[20] L. E. Sadler, J. M. Higbie, S. R. Leslie, M. Vengalattore, and D. M. Stamper-Kurn, "Spontaneous symmetry breaking in a quenched ferromagnetic spinor Bose-Einstein condensate," Nature (London) 443, 312 (2006).

[21] K. M. Mertes, J. W. Merrill, R. Carretero-González, D. J. Frantzeskakis, P. G. Kevrekidis, and D. S. Hall, "Nonequilibrium dynamics and superfluid ring excitations in binary BoseEinstein condensates," Phys. Rev. Lett. 99, 190402 (2007).

[22] R. P. Anderson, C. Ticknor, A. I. Sidorov, and B. V. Hall, "Spatially inhomogeneous phase evolution of a twocomponent Bose-Einstein condensate," Phys. Rev. A 80, 023603 (2009).

[23] Satoshi Tojo, Yoshihisa Taguchi, Yuta Masuyama, Taro Hayashi, Hiroki Saito, and Takuya Hirano, "Controlling phase separation of binary Bose-Einstein condensates via mixedspin-channel Feshbach resonance," Phys. Rev. A 82, 033609 (2010).

[24] S. B. Papp, J. M. Pino, and C. E. Wieman, "Tunable miscibility in a dual-species Bose-Einstein condensate," Phys. Rev. Lett. 101, 040402 (2008).

[25] S. Händel, T. P. Wiles, A. L. Marchant, S. A. Hopkins, C. S. Adams, and S. L. Cornish, "Magnetic merging of ultracold atomic gases of ${ }^{85} \mathrm{Rb}$ and ${ }^{87} \mathrm{Rb}$," Phys. Rev. A 83, 053633 (2011).

[26] Seiji Sugawa, Rekishu Yamazaki, Shintaro Taie, and Yoshiro Takahashi, "Bose-Einstein condensate in gases of rare atomic species," Phys. Rev. A 84, 011610 (2011).

[27] Kazuki Sasaki, Naoya Suzuki, Daisuke Akamatsu, and Hiroki Saito, "Rayleigh-taylor instability and mushroom-pattern formation in a two-component bose-einstein condensate," Phys. Rev. A 80, 063611 (2009).

[28] S. Gautam and D. Angom, "Rayleigh-taylor instability in binary condensates," Phys. Rev. A 81, 053616 (2010).

[29] S Gautam and D Angom, "Ground state geometry of binary condensates in axisymmetric traps," Journal of Physics B: Atomic, Molecular and Optical Physics 43, 095302 (2010).

[30] Shai Ronen, John L. Bohn, Laura Elisa Halmo, and Mark Edwards, "Dynamical pattern formation during growth of a dualspecies Bose-Einstein condensate," Phys. Rev. A 78, 053613 (2008).

[31] M. A. Hoefer, J. J. Chang, C. Hamner, and P. Engels, "Dark-dark solitons and modulational instability in miscible two-component Bose-Einstein condensates," Phys. Rev. A 84, 041605 (2011).
[32] C. Hamner, J. J. Chang, P. Engels, and M. A. Hoefer, "Generation of dark-bright soliton trains in superfluid-superfluid counterflow," Phys. Rev. Lett. 106, 065302 (2011).

[33] S. De, D. L. Campbell, R. M. Price, A. Putra, Brandon M. Anderson, and I. B. Spielman, "Quenched binary Bose-Einstein condensates: Spin-domain formation and coarsening," Phys. Rev. A 89, 033631 (2014).

[34] Tin-Lun Ho and V. B. Shenoy, "Binary mixtures of bose condensates of alkali atoms," Phys. Rev. Lett. 77, 3276-3279 (1996).

[35] P. Ao and S. T. Chui, "Binary bose-einstein condensate mixtures in weakly and strongly segregated phases," Phys. Rev. A 58, 4836-4840 (1998).

[36] S Gautam and D Angom, "Phase separation of binary condensates in harmonic and lattice potentials," Journal of Physics B: Atomic, Molecular and Optical Physics 44, 025302 (2011).

[37] Arko Roy and D. Angom, "Thermal suppression of phase separation in condensate mixtures," Phys. Rev. A 92, 011601 (2015).

[38] Soumik Bandyopadhyay, Arko Roy, and D. Angom, "Dynamics of phase separation in two-species bose-einstein condensates with vortices," Phys. Rev. A 96, 043603 (2017).

[39] S Gautam, P Muruganandam, and D Angom, "Coreless vortex dipoles and trapped droplets in phase-separated binary condensates," Journal of Physics B: Atomic, Molecular and Optical Physics 45, 055303 (2012).

[40] S. Gautam, P. Muruganandam, and D. Angom, "Formation and stability of coreless vortex dipoles in phase-separated binary condensates," Physics Letters A 377, 378 - 386 (2013).

[41] Arko Roy and D. Angom, "Fluctuation- and interactioninduced instability of dark solitons in single and binary condensates," Phys. Rev. A 90, 023612 (2014).

[42] Pekko Kuopanportti, Soumik Bandyopadhyay, Arko Roy, and D. Angom, "Splitting of singly and doubly quantized composite vortices in two-component bose-einstein condensates," Phys. Rev. A 100, 033615 (2019).

[43] Yujiro Eto, Masahiro Takahashi, Keita Nabeta, Ryotaro Okada, Masaya Kunimi, Hiroki Saito, and Takuya Hirano, "Bouncing motion and penetration dynamics in multicomponent Bose-Einstein condensates," Phys. Rev. A 93, 033615 (2016).

[44] Yujiro Eto, Masahiro Takahashi, Masaya Kunimi, Hiroki Saito, and Takuya Hirano, "Nonequilibrium dynamics induced by miscible-immiscible transition in binary BoseEinstein condensates," New J. Phys. 18, 073029 (2016).

[45] Arko Roy, S. Gautam, and D. Angom, "Goldstone modes and bifurcations in phase-separated binary condensates at finite temperature," Phys. Rev. A 89, 013617 (2014).

[46] K. Suthar, Arko Roy, and D. Angom, "Fluctuation-driven topological transition of binary condensates in optical lattices," Phys. Rev. A 91, 043615 (2015).

[47] A. Roy, S. Gautam, and D. Angom, "Evolution of goldstone mode in binary condensate mixtures," The European Physical Journal Special Topics 224 (2015), 10.1140/epjst/e201502388-8.

[48] K. Suthar and D. Angom, "Optical-lattice-influenced geometry of quasi-two-dimensional binary condensates and quasiparticle spectra,” Phys. Rev. A 93, 063608 (2016).

[49] K. Suthar and D. Angom, "Characteristic temperature for the immiscible-miscible transition of binary condensates in optical lattices," Phys. Rev. A 95, 043602 (2017).

[50] Sukla Pal, Arko Roy, and D Angom, "Bifurcations, stability and mode evolution in segregated quasi- $2 \mathrm{~d}$ condensate mixtures," Journal of Physics B: Atomic, Molecular and Optical 
Physics 50, 195301 (2017).

[51] Sukla Pal, Arko Roy, and D Angom, "Collective modes in multicomponent condensates with anisotropy," Journal of Physics B: Atomic, Molecular and Optical Physics 51, 085302 (2018).

[52] E. Nicklas, M. Karl, M. Höfer, A. Johnson, W. Muessel, H. Strobel, J. Tomkovič, T. Gasenzer, and M. K. Oberthaler, "Observation of scaling in the dynamics of a strongly quenched quantum gas," Phys. Rev. Lett. 115, 245301 (2015).

[53] Peter K. Molony, Philip D. Gregory, Zhonghua Ji, Bo Lu, Michael P. Köppinger, C. Ruth Le Sueur, Caroline L. Blackley, Jeremy M. Hutson, and Simon L. Cornish, "Creation of ultracold ${ }^{87} \mathrm{Rb}^{133} \mathrm{Cs}$ molecules in the rovibrational ground state," Phys. Rev. Lett. 113, 255301 (2014).

[54] Mingyang Guo, Bing Zhu, Bo Lu, Xin Ye, Fudong Wang, Romain Vexiau, Nadia Bouloufa-Maafa, Goulven Quéméner, Olivier Dulieu, and Dajun Wang, "Creation of an ultracold gas of ground-state dipolar ${ }^{23} \mathrm{Na}^{87} \mathrm{Rb}$ molecules," Phys. Rev. Lett. 116, 205303 (2016).

[55] Sebastian A. Will, Jee Woo Park, Zoe Z. Yan, Huanqian Loh, and Martin W. Zwierlein, "Coherent microwave control of ultracold ${ }^{23} \mathrm{Na}^{40} \mathrm{~K}$ molecules," Phys. Rev. Lett. 116, 225306 (2016).

[56] J. Catani, L. De Sarlo, G. Barontini, F. Minardi, and M. Inguscio, "Degenerate Bose-Bose mixture in a three-dimensional optical lattice,” Phys. Rev. A 77, 011603 (2008).

[57] Bryce Gadway, Daniel Pertot, René Reimann, and Dominik Schneble, "Superfluidity of interacting bosonic mixtures in optical lattices," Phys. Rev. Lett. 105, 045303 (2010).

[58] Ehud Altman, Walter Hofstetter, Eugene Demler, and Mikhail D Lukin, "Phase diagram of two-component bosons on an optical lattice," New Journal of Physics 5, 113 (2003).

[59] Guang-Hong Chen and Yong-Shi Wu, "Quantum phase transition in a multicomponent Bose-Einstein condensate in optical lattices," Phys. Rev. A 67, 013606 (2003).

[60] A. B. Kuklov and B. V. Svistunov, "Counterflow superfluidity of two-species ultracold atoms in a commensurate optical lattice," Phys. Rev. Lett. 90, 100401 (2003).

[61] Anatoly Kuklov, Nikolay Prokof'ev, and Boris Svistunov, "Commensurate two-component bosons in an optical lattice: Ground state phase diagram," Phys. Rev. Lett. 92, 050402 (2004).

[62] A. Trautmann, P. Ilzhöfer, G. Durastante, C. Politi, M. Sohmen, M. J. Mark, and F. Ferlaino, "Dipolar quantum mixtures of erbium and dysprosium atoms," Phys. Rev. Lett. 121, 213601 (2018).

[63] Tapan Mishra, Ramesh V. Pai, and B. P. Das, "Phase separation in a two-species Bose mixture," Phys. Rev. A 76, 013604 (2007).

[64] Fei Zhan and Ian P. McCulloch, "Comment on "Phase separation in a two-species Bose mixture"," Phys. Rev. A 89, 057601 (2014).

[65] Wei Wang, Vittorio Penna, and Barbara Capogrosso-Sansone, "Analysis and resolution of the ground-state degeneracy of the two-component Bose-Hubbard model," Phys. Rev. E 90, 022116 (2014).

[66] A. Isacsson, Min-Chul Cha, K. Sengupta, and S. M. Girvin, "Superfluid-insulator transitions of two-species bosons in an optical lattice," Phys. Rev. B 72, 184507 (2005).

[67] M. Iskin, "Strong-coupling expansion for the two-species bose-hubbard model," Phys. Rev. A 82, 033630 (2010).

[68] S. Anufriiev and T. A. Zaleski, "Multicriticality and interaction-induced first-order phase transitions in mixtures of ultracold bosons in an optical lattice,” Phys. Rev. A 94, 043613 (2016).

[69] Massimo Boninsegni, "Phase separation in mixtures of hard core bosons," Phys. Rev. Lett. 87, 087201 (2001).

[70] Ramesh V. Pai, Jamshid Moradi Kurdestany, K. Sheshadri, and Rahul Pandit, "Bose-Hubbard models in confining potentials: Inhomogeneous mean-field theory," Phys. Rev. B 85, 214524 (2012)

[71] B. Capogrosso-Sansone, C. Trefzger, M. Lewenstein, P. Zoller, and G. Pupillo, "Quantum phases of cold polar molecules in 2d optical lattices," Phys. Rev. Lett. 104, 125301 (2010).

[72] T. Flottat, L. de Forges de Parny, F. Hébert, V. G. Rousseau, and G. G. Batrouni, "Phase diagram of bosons in a twodimensional optical lattice with infinite-range cavity-mediated interactions," Phys. Rev. B 95, 144501 (2017).

[73] M. Iskin, "Route to supersolidity for the extended bosehubbard model," Phys. Rev. A 83, 051606 (2011).

[74] Kwai-Kong Ng and Yung-Chung Chen, "Supersolid phases in the bosonic extended hubbard model," Phys. Rev. B 77, 052506 (2008).

[75] Daisuke Yamamoto and Ippei Danshita, "Stability of superflow in supersolid phases of lattice bosons with dipoledipole interaction," Journal of Physics: Conference Series 273, 012020 (2011).

[76] Massimo Boninsegni and Nikolay V. Prokof'ev, "Colloquium: Supersolids: What and where are they?" Rev. Mod. Phys. 84, 759-776 (2012).

[77] Léonard Julian, Morales Andrea, Zupancic Philip, Esslinger Tilman, and Donner Tobias, "Supersolid formation in a quantum gas breaking a continuous translational symmetry," Nature 543, 87 (2017).

[78] Yoshihito Kuno, Keita Suzuki, and Ikuo Ichinose, "Superfluidity and solid order in a two-component bose gas with dipolar interactions in an optical lattice," Phys. Rev. A 90, 063620 (2014).

[79] Ryan M. Wilson, Wilbur E. Shirley, and Stefan S. Natu, "Anomalous supersolidity in a weakly interacting dipolar bose mixture on a square lattice," Phys. Rev. A 93, 011605 (2016).

[80] B. Damski, L. Santos, E. Tiemann, M. Lewenstein, S. Kotochigova, P. Julienne, and P. Zoller, "Creation of a dipolar superfluid in optical lattices," Phys. Rev. Lett. 90, 110401 (2003).

[81] Marek Trippenbach, Krzysztof Góral, Kazimierz Rzazewski, Boris Malomed, and Y B Band, "Structure of binary boseeinstein condensates," Journal of Physics B: Atomic, Molecular and Optical Physics 33, 4017 (2000).

[82] D. S. Rokhsar and B. G. Kotliar, "Gutzwiller projection for bosons," Phys. Rev. B 44, 10328 (1991).

[83] K. Sheshadri, H. R. Krishnamurthy, R. Pandit, and T. V. Ramakrishnan, "Superfluid and insulating phases in an interacting-boson model: Mean-field theory and the RPA," EPL 22, 257 (1993).

[84] Rukmani Bai, Soumik Bandyopadhyay, Sukla Pal, K. Suthar, and D. Angom, "Bosonic quantum hall states in single-layer two-dimensional optical lattices," Phys. Rev. A 98, 023606 (2018).

[85] Sukla Pal, Rukmani Bai, Soumik Bandyopadhyay, K. Suthar, and D. Angom, "Enhancement of the bose glass phase in the presence of an artificial gauge field," Phys. Rev. A 99, 053610 (2019).

[86] K. Suthar, Hrushikesh Sable, Rukmani Bai, Soumik Bandyopadhyay, Sukla Pal, and D. Angom, "Supersolid phase of the extended bose-hubbard model with an artificial gauge field," 
Phys. Rev. A 102, 013320 (2020).

[87] Yijun Tang, Wil Kao, Kuan-Yu Li, and Benjamin L. Lev, "Tuning the dipole-dipole interaction in a quantum gas with a rotating magnetic field," Phys. Rev. Lett. 120, 230401 (2018).

[88] Stefano Giovanazzi, Axel Görlitz, and Tilman Pfau, "Tuning the dipolar interaction in quantum gases," Phys. Rev. Lett. 89, 130401 (2002).

[89] D. van Oosten, P. van der Straten, and H. T. C. Stoof, "Quantum phases in an optical lattice," Phys. Rev. A 63, 053601 (2001).

[90] M. Iskin and J. K. Freericks, "Strong-coupling perturbation theory for the extended bose-hubbard model," Phys. Rev. A 79, 053634 (2009).

[91] L. de Forges de Parny, F. Hébert, V. G. Rousseau, R. T. Scalettar, and G. G. Batrouni, "Ground-state phase diagram of spin$\frac{1}{2}$ bosons in a two-dimensional optical lattice," Phys. Rev. B 84, 064529 (2011).

[92] Yasuyuki Kato, Daisuke Yamamoto, and Ippei Danshita, "Quantum tricriticality at the superfluid-insulator transition of binary bose mixtures," Phys. Rev. Lett. 112, 055301 (2014).

[93] D.-S. Lühmann, "Cluster Gutzwiller method for bosonic lattice systems," Phys. Rev. A 87, 043619 (2013).

[94] K. W. Mahmud, E. N. Duchon, Y. Kato, N. Kawashima, R. T. Scalettar, and N. Trivedi, "Finite-temperature study of bosons in a two-dimensional optical lattice," Phys. Rev. B 84, 054302 (2011).

[95] L. de Forges de Parny, F. Hébert, V. G. Rousseau, and G. G. Batrouni, "Finite temperature phase diagram of spin$1 / 2$ bosons in two-dimensional optical lattice," Eur. Phys. J. B
85, 169 (2012).

[96] Kean Loon Lee, Nils B. Jørgensen, I-Kang Liu, Lars Wacker, Jan J. Arlt, and Nick P. Proukakis, "Phase separation and dynamics of two-component bose-einstein condensates," Phys. Rev. A 94, 013602 (2016).

[97] Hiromitsu Takeuchi, Naoya Suzuki, Kenichi Kasamatsu, Hiroki Saito, and Makoto Tsubota, "Quantum kelvin-helmholtz instability in phase-separated two-component bose-einstein condensates," Phys. Rev. B 81, 094517 (2010).

[98] E. Lundh and J.-P. Martikainen, "Kelvin-helmholtz instability in two-component bose gases on a lattice," Phys. Rev. A 85, 023628 (2012).

[99] Jakub Zakrzewski, "Mean-field dynamics of the superfluidinsulator phase transition in a gas of ultracold atoms," Phys. Rev. A 71, 043601 (2005).

[100] D. L. Kovrizhin, G. Venketeswara Pai, and S. Sinha, "Density wave and supersolid phases of correlated bosons in an optical lattice," Europhys. Lett. 72, 162-168 (2005).

[101] Konstantin V. Krutitsky, Jonas Larson, and Maciej Lewenstein, "Dark solitons near the mott-insulator-superfluid phase transition," Phys. Rev. A 82, 033618 (2010).

[102] Konstantin V. Krutitsky and Patrick Navez, "Excitation dynamics in a lattice bose gas within the time-dependent gutzwiller mean-field approach," Phys. Rev. A 84, 033602 (2011).

[103] Takuya Saito, Ippei Danshita, Takeshi Ozaki, and Tetsuro Nikuni, "Detecting the superfluid critical momentum of bose gases in optical lattices through dipole oscillations," Phys. Rev. A 86, 023623 (2012). 